Consiglio Nazionale delle Ricerche

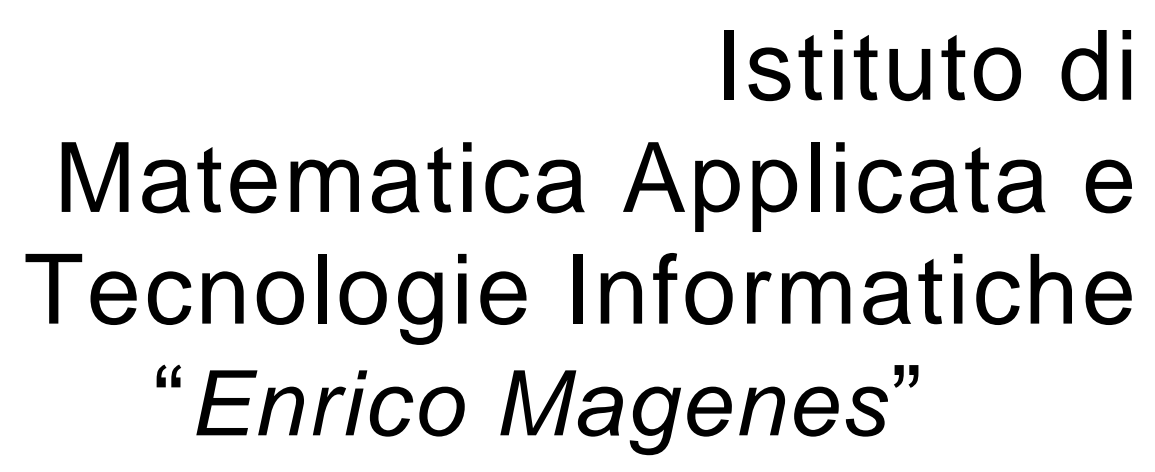

PUBBLICAZIONI

M.S. Pauletti, M. Martinelli, N. Cavallini, P. Antolín

IGATOOLS: AN ISOGEOMETRIC ANALYSIS LIBRARY

3PV14/1/0 
C.N.R. - Istituto di Matematica Applicata e Tecnologie Informatiche "Enrico Magenes"

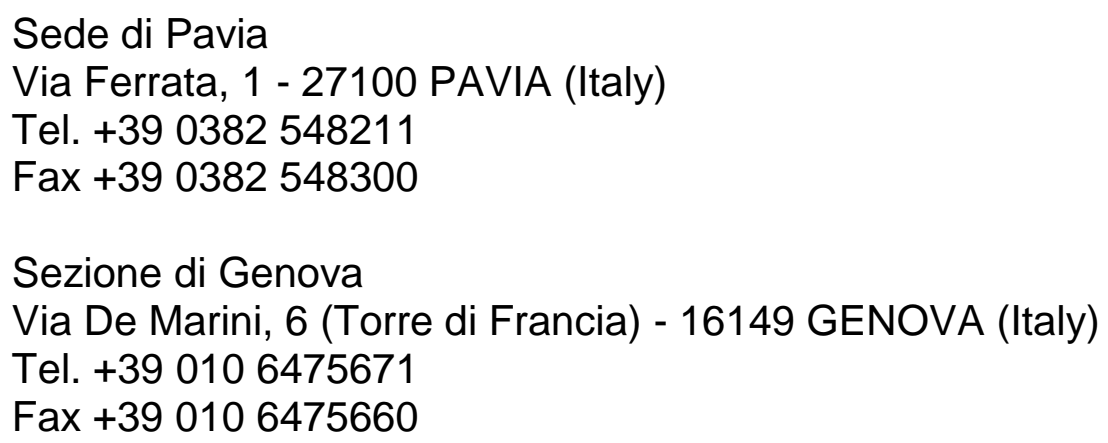

URL: http://www.imati.cnr.it 


\title{
IGATOOLS: AN ISOGEOMETRIC ANALYSIS LIBRARY
}

\author{
M. SEBASTIAN PAULETTI*, MASSIMILIANO MARTINELLI ${ }^{\dagger},{\text { NICOLA CAVALLINI }{ }^{\ddagger}, \text { AND PABLO ANTOLÍN }}^{\S}$
}

\begin{abstract}
We present a novel mathematically faithful object oriented design for a general purpose isogeometric library and introduce a high quality open source implementation of it, igatools (http://code.google.com/p/igatools). The library uses advanced programming techniques and supports dimension independent programming. It includes support for manifolds and isogeometric elements of the h-div and h-curl type. To illustrate the flexibility and power of the library we present some example applications including surfaces, fluid and elasticity.
\end{abstract}

Key words. isogeometric analysis, software library, open source, object oriented, generic programming, dimension independent, B-splines, NURBS, h div, h curl, CAD integration

AMS subject classifications. 65N30, 97N80, 97N40, 68N19, 65D07

1. Introduction. Inspired by the desire to unify the fields of computer aided geometrical design (CAGD) and the finite element method (FEM), the visionary work 30 introduced a technique for the discretization of partial differential equations dubbed isogeometric analysis (IGA). In this work, any numerical method fitting the isogeometric analysis framework will be referred to as an isogeometric method (IgM). The mainly advertised feature of an IgM has been the ability to describe exactly CAGD type geometries. This is so because the method proposes to use the same type of spaces to represent the geometry and the shape functions (mostly non uniform rational B-splines). But in addition to exact representation of CAGD geometries the use of B-spline functions allows global smoothness beyond the classical $C^{0}$ continuity of standard finite elements: this permits the design of novel numerical schemes that would be extremely difficult to obtain with standard finite elements. Isogeometric methods have been summarized in a recent book [19] and studied in [16, 17]. They have been successfully used in applications such as fluid [6, 7, 24], structural mechanics [4, 33, 20].

After a decade of research in the area, with many successes, some issues (that require further research) and many promising ideas, it is time -at least in the research community- to have an open source modernly designed isogeometric software library. Which should be capable of dealing with the current understanding of an IgM while providing the flexibility to adapt to the future evolution of the IgM. And not less important, to justify the time invested in its learning, it should follow high quality standards in design and development. Unfortunately, we did not find an isogeometric library with the features and quality standards of our expectations. This need and the scientific environment at Pavia provided the supporting framework for us to undertake the first step toward the creation of such software. Pavia enjoined the early stages on the development of the isogeometric concept. Several scientists in mathematics and engineering contributed with their work to the theoretical and computational development of isogeometric analysis. For several years GeoPDEs [21] (an easy to use MATLAB toolkit) has "propelled" the dissemination of the concept in its early stages partially paving the road for our work.

The main purpose of this work is to present an object oriented design for an isogeometric software library and introduce a high quality implementation of it, igatools. igatools is a general purpose open source library to solve partial differential equations using isogeometric type spaces. Unlike most academic

*Instituto de Matemática Aplicada del Litoral (IMAL). Consejo Nacional de Investigaciones científicas y técnicas (CONICET). Santa Fe, Argentina. Partially supported by European Research Council through the FP7 Ideas Starting Grant 205004 GeoPDEs and Agencia Nacional de Promoción Científica y Tecnológica, through grant PICT-2008-0622 (Argentina).

${ }^{\dagger}$ Istituto di Matematica Applicata e Tecnologie Informatiche (IMATI). Consiglio Nazionale delle Ricerche (CNR). Pavia, Italy. Partially supported by Hutchinson-Total under contract TOTAL DS-2753 and FIRB "Futuro in Ricerca" RBFR08CZ0S.

${ }_{\ddagger}^{\ddagger}$ Dipartimento di Matematica "F. Casorati". Università degli Studi di Pavia. Via Ferrata 1, 27100 Pavia, Italy. Pavia, Italy. Supported by the TERRIFIC project, European Communitys Seventh Framework Programme, Grant Agreement 284981 Call FP7-2011-NMP-ICT-FoF.

$\S$ Dipartimento di Ingegneria Civile ed Architettura. Università degli Studi di Pavia. Via Ferrata 3, 27100 Pavia, Italy. Partially supported by Hutchinson-Total under contract TOTAL DS-2753 and by European Research Council through the FP7 Ideas Starting Grant 259229 ISOBIO. 
software, that start as a tool to solve the specific applications of a small research group, igatools was conceived from the beginning to provide useful tools to aid in the approximation to a wide range of possible applications.

The design of igatools encapsulates the mathematical concepts of an isogeometric method into objects and map their relations into interaction between these objects. We tried hard to attain the simplest possible design that is faithful to the mathematics behind. Figure 3.1 shows a blueprint of the basic design. As a guiding rule to validate the design we use the following: "if an awkward code is needed to perform a task consistent with the mathematics of the problem then there is a problem with the design of the library". Desirable consequences of such a design are improved maintainability, as it produces a code that reads closer to the human conception of the problem, and flexibility for extensions, as adding a new feature coming from the mathematics of the problem should face no major restrictions.

A good design is paramount for a software library to transcend the original group of developers. But it is also necessary to carefully plan and execute the structure and documentation of the code, as well as the infrastructure to support its development. In igatools we have considered all these principles. The library is implemented in $\mathrm{C}++11$ [32, 9, its design is object oriented with extensive use of generic programming techniques that allows among other advantages to obtain code that is dimension independent while suppressing the overhead of virtual functions. The development environment follows the high quality standards of today software engineering: we use a platform independent configuration system (CMake), an automatic test suite (CTest/CDash), a version control system (git), a bug tracking system (Trac), an in-source documentation system (Doxygen), a user discussion forum and an on-line tutorial. On top of the novel object oriented design that it implements, igatools provides practical useful features such as the possibility of floating-point types of different precision, integration with CAGD modelers, and parallel support for shared (multi-threading) and distributed (MPI) memory systems.

The IgM is closely related to the well known FEM, they are both Galerkin methods that provide an specific way of constructing the basis functions of the discrete approximating spaces. Some finite element spaces can be generated with the IgM (Lagrange elements on quadrilaterals for example). In a loose sense an IgM can be interpreted as a generalized FEM where the basis functions are more regular. But this interpretation starts to diverge as a closer look is taken onto the details, for example even though the Lagrange spaces are the same, the basis functions generated using the FEM are different from the ones of the IgM. It is our view that even though a finite element code could be adapted to accept an isogeometric plugin 13, this approach presents some shortcoming. The popular well-maintained, modernly designed and stable FE libraries are so tailored for the FEM that using them for and IgM makes some forced interpretation of a few concepts. In fact, we would need to define an isogeometric counterpart for the finite element triple or the unstructured triangulation. It is our opinion that forcing $\operatorname{IgM}$ concepts to fit into a FEM design, without existing a true matching between concepts, will lead to a shady design affecting clarity, maintainability, efficiency and extensibility of the code. Still we should not forget the similarities these methods share. Our IgM design is inspired by these similarities as well as these differences to produce a software that suites an easy implementation of state of the art method and original techniques. In particular, we have absorbed many applicable concepts from an open source finite element library that seems to have survived the test of time, namely the deal. II library [2, 3].

This work is neither a manual nor a user guide for igatools. In fact, there are online documentation (more than hundreds of pages if printed) and a tutorial dedicated for these purposes. The main purpose is to present and explain a novel object oriented design for isogeometric analysis faithful to the mathematics of the IgM and introduce a high quality software implementation of it, igatools.

The outline for this work follows the ideal approach for designing a computer program (see [42) in three stages: analysis, design and programming. In the analysis we need to gain a clear understanding of the problem, in our case the IgM, this is what we do in $\$ 2$, where we describe the method from the viewpoint of designing a software library for it. The design is presented in $\$ 3$ where the key concepts of the IgM and their relations are identified and mapped into objects. Here we also detail the main classes implemented in igatools (the programming stage). Finally, in $\$ 6$ we present some common applications that our library can easily accommodate including the Poisson problem, Laplace-Beltrami on surfaces, a 
fluid application and an elasticity problem.

2. Isogeometric spaces. In order to design an isogeometric library, it is crucial first to achieve a clear understanding of what an isogeometric method is. Since its introduction what we call isogeometric method (IgM) has evolved to incorporate new concepts and ideas. The original emphasis when IGA was introduced was on the exact representation of the geometry and the requirement of the isoparametric paradigm to be satisfied. On one hand, new reference spaces beside NURBS has been introduced (Tsplines [5, 23, 15, 39], locally refined splines [22] and hierarchical splines [26, 27, 43]). On the other hand, the isoparametric constraint has been lately relaxed [18, 17, 16] to include mappings that are not necessarily in the reference space. We also support the relaxation of the exact domain geometry constraint. From the mathematical point of view, is not mandatory to have an exact representation of the domain (what is needed is an approximation of the geometry that leads to an error of the same order of the one that is due to the approximation order of the discrete space), but from an engineering point of view it is extremely useful to have an exact representation of the geometry: indeed with an exact representation, the geometry (and the grid built upon it) can be easily modified/manipulated (for instance in optimization loops) without the needs of re-meshing procedures. For the approximation of some vector-valued problems to be stable, it is known [11, 35, that the approximating spaces must satisfy some properties. Particular examples are the Raviar-Thomas and the edge elements. A more general mathematical framework in the language of differential forms and de Rham cohomology has been studied in [1] and applied in the context of isogeometric methods in [18, 17]. Our design supports this spaces through the use of a transformation type and the concept of an associated push-forward operator.

Thus in our design, we conceive an isogeometric method to be a method that incorporates most existing flavors of isogeometric techniques such as the ones described in the previous paragraph. But moreover, based on the fact that the design strives to be conceptually faithful to the mathematics, we expect the software to be flexible enough to adapt to and encourage the creation of new methods that are unknown at the moment.

In this section we give a brief overview of the isogeometric method, B-splines and NURBS while introducing the notation used in this work. Details can be found in the standard bibliography, for example [38, 36, 19].

2.1. The isogeometric method. An IgM is a type of Galerkin method to approximate the solution of boundary value problems. To make the discussion more concrete let us consider a differential equation in a $d$-dimensional domain $\Omega \subset \mathbb{R}^{s}$, being $s$ the dimension of the embedding euclidean space and $s-d$ the codimension of the domain. More precisely, let $V$ be a Sobolev space (infinite dimensional) on $\Omega$, $\mathcal{A}: V \rightarrow V^{\prime}$ a differential operator and $f \in V^{\prime}$ a linear functional. The variational problem is to find $u \in V$ such that $\mathcal{A} u=f$. The Galerkin procedure approximates the solution $u$ by solving a similar problem in finite dimensional subspaces. The IgM (as well as the FEM) provides a recipe to construct these discrete spaces. More specifically, we define an IgM as one providing:

1. A reference space. Given a $d$-dimensional rectangle $\hat{\Omega} \subset \mathbb{R}^{d}$ that we call the reference domain, provides a specific way to construct a reference space $\hat{\mathbb{V}}(\hat{\Omega})$ of the B-spline type (see definition 2.1).

2. A deformation. Provides a smooth deformation $\boldsymbol{F}: \hat{\Omega} \rightarrow \mathbb{R}^{s}$ (with smooth inverse). We refer to $\boldsymbol{F}$ as the mapping and to $\Omega=\boldsymbol{F}(\hat{\Omega})$ as the physical domain.

3. A transformation type. This is a rule that specifies how to use the deformation to transform the functions in the reference space into the ones in the physical space. Some example besides direct composition are divergence and curl preserving transformations (cf. Table 2.1.

The mapping together with the transformation type define a push-forward operator ${ }^{1} P$ and this operator acts on the reference space to give the physical space $\mathbb{V}(\Omega)$ (see Figure 2.1 as $\mathbb{V}(\Omega)=\{\phi=$ $P(\hat{\phi})$ with $\hat{\phi} \in \hat{\mathbb{V}}(\hat{\Omega})\}$. We say that $\mathbb{V}(\Omega)$ is an isogeometric space and a function $u \in \mathbb{V}(\Omega)$, is generally referred to as a field.

\footnotetext{
${ }^{1}$ In the context of differential geometry $P$ would be called the pullback (as there is no distinction between reference and physical), for our setting it is more natural and clear to refer to $P$ as the push-forward of reference functions to physical functions.
} 


\begin{tabular}{cccc}
\hline h_grad & h_curl & h_div & 1_2 \\
\hline$\phi=\hat{\phi} \circ \boldsymbol{F}^{-1}$ & $\boldsymbol{u}=D \boldsymbol{F}^{-T}\left(\hat{\boldsymbol{u}} \circ \boldsymbol{F}^{-1}\right)$ & $\boldsymbol{v}=\frac{D \boldsymbol{F}}{\operatorname{det} D \boldsymbol{F}}\left(\hat{\boldsymbol{v}} \circ \boldsymbol{F}^{-1}\right)$ & $\varphi=\frac{\hat{\varphi} \circ \boldsymbol{F}^{-1}}{\operatorname{det} D \boldsymbol{F}}$ \\
\hline
\end{tabular}

Examples of different transformation types to obtain a physical discrete space $\mathbb{V}$ from a reference spline or NURBS space $\hat{\mathbb{V}}$ and a mapping $\boldsymbol{F}$. The table shows the transformation name given in the library and the formula that defines its push-forward operator.
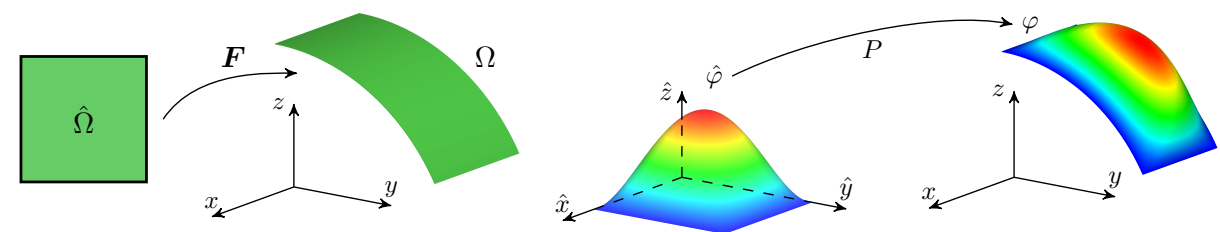

FIG. 2.1. The isogeometric method delivers a recipe to construct a discrete space by providing a reference space of the $B$-Spline type, a deformation $\boldsymbol{F}$ and a transformation type. The transformation type indicates how to use the deformation to define the push-forward operator $P$. The physical space $\mathbb{V}(\Omega)$ is obtained as the image of the reference space $\hat{\mathbb{V}}(\hat{\Omega})$ through $P$.

\subsection{B-splines and NURBS.}

2.2.1. Univariate B-spline. Given a non negative number $p$, a spline of degree $p$ on the interval $[a, b]$ is a real valued piecewise polynomial function of degree at most $p$ on each subinterval of $[a, b]$ determined by the partition $a=\zeta_{1}<\cdots<\zeta_{m}=b$. The $\zeta_{i}$ 's are called the knots and they form the knot vector $\boldsymbol{\zeta}=\left(\zeta_{1}, \ldots, \zeta_{m}\right)$. At each knot, the spline function is allowed to have a regularity that ranges from discontinuous $\left(C^{-1}\right)$ to $C^{p-1}$, this is usually indicated using the so called regularity vector $\boldsymbol{\alpha}=\left(\alpha_{1}, \ldots, \alpha_{m}\right)$ where $\alpha_{i} \in \mathbb{Z}$ and $-1 \leq \alpha_{i} \leq p-1$. It is sometimes convenient to encode both knot and regularity vectors into a single vector of repeated knots $\boldsymbol{\xi}=(\underbrace{\zeta_{1}, \ldots, \zeta_{1}}_{r_{1} \text { times }}, \underbrace{\zeta_{2}, \ldots, \zeta_{2}}_{r_{2} \text { times }}, \ldots, \underbrace{\zeta_{m}, \ldots, \zeta_{m}}_{r_{m} \text { times }})$, where $r_{i}=p-\alpha_{i}$, and $\boldsymbol{r}=\left(r_{1}, \ldots, r_{m}\right)$ is called the multiplicity vector. When the multiplicity of the first and last knots is $p+1$ we call $\boldsymbol{\xi}$ an open knot vector.

We define $S_{\boldsymbol{\xi}}^{p}$ to be the space of spline functions of degree $p$ subordinated to the knot vector with repetition $\boldsymbol{\xi}$. It is a well known result that the dimensionality of this space is $n=\sum_{i=1}^{m} r_{i}-p-1$. The classical Cox-de Boor recursive algorithm [36] allows to construct a basis for $S_{\boldsymbol{\xi}}^{p}$, known as the B-spline basis. We denote these basis functions by $B_{i}$ (see Figure 2.2). Some important properties of B-spline basis functions are:

(i) Non-negativity: each $B_{i}(x) \geq 0$ for all $x \in[a, b]$

(ii) Small support: each $B_{i}$ is non-zero only in $\left[\zeta_{i}, \zeta_{i+j}\right)$ with $1 \leq j \leq p+1$ and on each interval $\left[\zeta_{i}, \zeta_{i+1}\right)$ (with $i=1, \ldots, m-1$ ), only $p+1$ basis functions have non-zero values

(iii) Partition of unity: the set of basis functions $\left\{B_{1}, \ldots, B_{n}\right\}$ satisfies that $\sum_{i=1}^{n} B_{i}(x)=1$, for all $x \in[a, b]$ if $\boldsymbol{\xi}$ is an open knot vector.

REMARK 1 (Univariate B-spline evaluation). A convenient method to compute values and derivatives of univariate B-spline functions can be achieved using the so called Bézier extraction operator technique. This method was introduced by Borden et al. [13, as a local adaptation of the global Bézier decomposition algorithm [36, A5.6]. More precisely, as a B-spline function of degree $p$ restricted to the $k$-th interval $I_{k}=\left[\zeta_{k}, \zeta_{k+1}\right)$ is a polynomial of degree $p$, we can write this polynomial as a linear combination of the $p+1$ Bernstein polynomial basis functions $b_{j, p}$ of degree $p$ as

$$
\left.B_{i}(x)\right|_{\left[\zeta_{k}, \zeta_{k+1}\right)}=B_{k, i}(x)=\sum_{j=1}^{p+1} c_{i j}^{(k)} b_{j, p}\left(\frac{x-\zeta_{k}}{\zeta_{k+1}-\zeta_{k}}\right), \quad i=1, \ldots, p+1,
$$




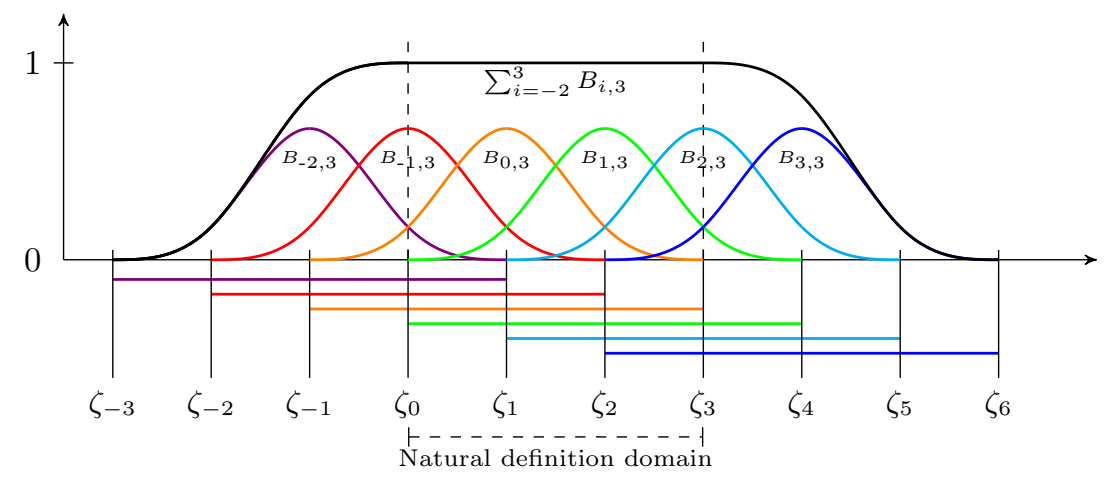

FIG. 2.2. One dimensional B-spline basis functions of degree 3 for a maximum regularity knot vector. As it can be seen, all the functions are non-negative; they span over four knot intervals (small support); on each interval only four function have non-zero values; and they form a partition of unity on the interval $\left[\zeta_{0}, \zeta_{3}\right]$.

with $b_{j, p}(t)=\left(\begin{array}{c}p \\ j-1\end{array}\right) t^{j-1}(1-t)^{p-j+1}$ for $0 \leq t \leq 1$ and $j=1, \ldots, p+1$. The coefficients $c_{i j}^{(k)}$ define the $k$-th interval matrix $C^{(k)}=\left(c_{i j}^{(k)}\right)$, referred to as the $k$-th Bézier extraction operator. A simple consequence of equation (2.1) is that the $m$-th derivative of a B-spline function can be written as linear combination of the $m$-th derivatives of the Bernstein polynomials with the same coefficients $c_{i j}^{(k)}$ as the function. More precisely,

$$
B_{k, i}^{(m)}(x)=\sum_{j=1}^{p+1} c_{i j}^{(k)} \frac{d^{m} b_{j, p}}{d \zeta^{m}}\left(\frac{x-\zeta_{k}}{\zeta_{k+1}-\zeta_{k}}\right)=\frac{1}{\left(\zeta_{k+1}-\zeta_{k}\right)^{m}} \sum_{j=1}^{p+1} c_{i j}^{(k)} b_{j, p}^{(m)}(t)
$$

with $t=\frac{x-\zeta_{k}}{\zeta_{k+1}-\zeta_{k}}$. This property leads to an efficient method to compute values and derivatives of univariate B-splines. More precisely, assume we have a spline space with $N$ intervals and we want to compute the values and derivatives of the B-spline functions using the same quadrature scheme on each interval. According to equation $(2.2)$ we need to compute the matrices $C^{(k)}\left(N\right.$ times) and $b_{j, p}^{(m)}$ at the unit quadrature points (one time, independent of $N$ ). With this information pre-computed we can compute all the required values and derivatives of the B-spline functions over all the intervals. Thus making this evaluation method efficient and convenient, in the context of an IgM.

2.2.2. Multivariate B-splines. The univariate spline spaces can be used to generate multidimensional spline spaces through tensor product multiplication. More precisely, given a positive integer $d$ we consider the spline spaces $S_{\boldsymbol{\xi}_{i}}^{p_{i}}\left(\left[a_{i}, b_{i}\right]\right)$ for $i=1, \ldots, d$ and define the multivariate spline space $S_{\boldsymbol{\xi}_{1}, \ldots, \boldsymbol{\xi}_{d}}^{p_{1}, \ldots, p_{d}}(\hat{\Omega})=S_{\boldsymbol{\xi}_{1}}^{p_{1}}\left(\left[a_{1}, b_{1}\right]\right) \otimes \ldots \otimes S_{\boldsymbol{\xi}_{d}}^{p_{d}}\left(\left[a_{d}, b_{d}\right]\right)$, where $\hat{\Omega}$ is the hyper-rectangle $\left[a_{1}, b_{1}\right] \times \cdots \times\left[a_{d}, b_{d}\right]$. In this case the multivariate B-Spline basis functions are

$$
B_{i_{1}, \ldots, i_{d}}\left(x_{1}, \ldots, x_{d}\right)=B_{i_{1}}^{1}\left(x_{1}\right) \ldots B_{i_{d}}^{d}\left(x_{d}\right)
$$

with $B_{j}^{i} \in S_{\boldsymbol{\xi}_{i}}^{p_{i}}$ for $j=1, \ldots, n_{i}$ being the B-spline basis of the univariate spaces. The dimension of the space is $n=\prod_{i=1}^{d} n_{i}$.

To simplify the notation in the multidimensional setting we define $\boldsymbol{p}=\left(p_{1}, \ldots, p_{d}\right)$ to be the vector of degrees and $\overrightarrow{\boldsymbol{\xi}}=\left(\boldsymbol{\xi}_{1}, \ldots, \boldsymbol{\xi}_{d}\right)$ the vector of knot vectors with repetition. Then we can write $S_{\overrightarrow{\boldsymbol{\xi}}}^{\boldsymbol{p}}(\hat{\Omega})$ instead of $S_{\boldsymbol{\xi}_{1}, \ldots, \boldsymbol{\xi}_{d}}^{p_{1}, \ldots, p_{d}}(\hat{\Omega})$. Similarly, we consider a vector index like $\boldsymbol{i}=\left(i_{1}, \ldots, i_{d}\right)$ for tensor product structures such as basis functions or elements, or $\boldsymbol{m}=\left(m_{1}, \ldots, m_{d}\right)$ as a derivative multi-index. Thus we can write $D^{\boldsymbol{m}} B_{\boldsymbol{i}}(\boldsymbol{x})$ as a short notation for $\frac{\partial^{m} B_{i_{1}, \ldots, i_{d}}}{\partial x_{1}^{m_{1}} \ldots \partial x_{d}^{m d}}\left(x_{1}, \ldots, x_{d}\right)$, with $m=|\boldsymbol{m}|$. Associated to a vector of knot vectors $\zeta$ we define the grid (or mesh, or cartesian grid) $\hat{\mathcal{Q}}=\left\{\hat{Q}_{i_{1}, \ldots, i_{d}}=\left[\zeta_{i_{1}}^{1}, \zeta_{i_{1}+1}^{1}\right] \times \cdots \times\right.$ $\left[\zeta_{i_{d}}^{d}, \zeta_{i_{d}+1}^{d}\right]$ with $1 \leq i_{j} \leq m_{j}$ and $\left.1 \leq j \leq d\right\}$, and its members $\hat{Q}_{i}$ are called the elements. 
One way igatools exploits the computational benefits of tensor products and B-splines is stated in the following remark:

REMARK 2 (Uniform computation of any order derivatives). The tensor product structure of a multivariate B-spline together with the Bezier extraction technique provide the foundation to an algorithm that translates to a single piece of code capable of computing any order of derivatives for multivariate B-spline functions. To see this consider the multi-index $\boldsymbol{m}$ and differentiate 2.3 from where it follows that $D^{\boldsymbol{m}} B_{\boldsymbol{i}}(\boldsymbol{x})=\prod_{j=1}^{d}\left(B_{i_{j}}^{j}\right)^{\left(m_{j}\right)}\left(x_{j}\right)$. This formula can be implemented with a single code for any size of $m$ (whether 1 or 50 ). If the univariate derivatives $\left(B_{i_{j}}^{j}\right)^{\left(m_{j}\right)}$, required by the formula, can be easily precomputed this provides a uniform and efficient code that can compute multivariate B-splines derivatives of any order. And this is the case as we have shown in Remark 1. This level of computational simplicity and efficiency is proper of B-splines and cannot be achieved with NURBS functions.

2.2.3. Non uniform rational B-splines (NURBS). NURBS functions are quotients of spline functions, thus a subspace of the rational functions. A NURBS space is constructed from a spline space $S_{\vec{\xi}}^{p}(\hat{\Omega})$ and a given strictly positive weight function $\omega \in S_{\vec{\xi}}^{p}(\hat{\Omega})$ as follows

$$
N_{\overrightarrow{\boldsymbol{\xi}}}^{\boldsymbol{p}}(\hat{\Omega})=N_{\boldsymbol{\xi}_{1}, \ldots, \boldsymbol{\xi}_{d}}^{p_{1}, \ldots, p_{d}}(\hat{\Omega})=\left\{\frac{\phi}{\omega}: \phi \in S_{\vec{\xi}}^{\boldsymbol{p}}(\hat{\Omega})\right\}
$$

NURBS are a fundamental tool in computer graphics. In the original isogeometric method the physical domain (cf. 2.1 ) is always a NURBS deformation. Without disregarding its ubiquitous use in the CAGD community (for their exact representation of conics) and the historical role in the spread of IGA into a broader community, it is important to remark that the approximation properties of a NURBS space $N_{\vec{\xi}}^{p}(\hat{\Omega})$ is not better than the one of its associated spline space $S_{\vec{\xi}}^{p}(\hat{\Omega})$. With the addition that spline spaces enjoy important theoretical and computational properties that NURBS spaces do not (for example, a spline space is closed under the differentiation operation, while a NURBS space is not).

2.2.4. Tensor-valued multivariate B-splines. Most interesting problems in applications involve vector or tensor valued quantities or even systems of them. A tensor-valued spline space is one where each component function belongs to a scalar-valued multivariate spline space as described in $\$ 2.2 .2$. Given the integers $s>0$ and $k \geq 0$ let $\mathcal{T}^{k}\left(\mathbb{R}^{s}\right)$ be the space of rank $k$ tensors over the vector space $\mathbb{R}^{s}$. Given $s^{k}$ scalar-valued spline spaces $S_{\boldsymbol{i}}=S_{\overrightarrow{\boldsymbol{\xi}}_{\boldsymbol{i}}}^{\boldsymbol{p}_{\boldsymbol{i}}}(\hat{\Omega})$ where $\boldsymbol{i}=\left(i_{1}, \ldots, i_{k}\right)$ is a tensor index with $1 \leq i_{j} \leq s$, we define the tensor valued spline space $\boldsymbol{S}^{s, k}$ to be the set of all tensor valued functions $\phi: \hat{\Omega} \rightarrow \mathcal{T}^{k}\left(\mathbb{R}^{s}\right)$ such that $\phi_{i_{1}, \ldots, i_{k}} \in S_{i_{1}, \ldots, i_{k}}(\hat{\Omega})$. In particular we get scalar, vector and tensor valued spaces if $k$ equals 0,1 or 2 respectively. We can similarly define a tensor valued NURBS space.

Definition 2.1 (B-spline type spaces). We use the term B-spline type space to refer to either a spline or NURBS space. And in general to refer to any space that can be generated by some operation involving B-spline basis functions.

2.3. Multipatch. So far we have considered a physical domain $\Omega$ that is the smooth deformation of a hyper-rectangle $\hat{\Omega}$. For some geometries this is enough but in some cases, even for simple geometries, such as the one in Figure 2.3, this is not possible. The natural solution used in CAGD is to construct the physical domain as a gluing of the box-like domains (the patches). Proceeding in a similar way one would glue together the physical spaces over each patch to obtain a physical space in the physical geometry.

The library allows conforming $C^{0}$ gluing of patches, and we are currently conducting the research work on gluing with higher regularity. The latter being an open issue that goes beyond the scope of this paper.

3. IgM software design. The main theoretical contribution of this work is a novel (the first one as far as we know) object oriented software design for the IgM. The main practical contribution is a high quality implementation of this design in the open source library igatools. Figure 3.1 shows a top level blueprint of this design that will be described in this section. Starting by a global overview in $\$ 3.1$ we continue to emphasize two important considerations that contributed to the design: a discussion on the similarities and differences with the FEM and a generic programming model for dimension and rank 


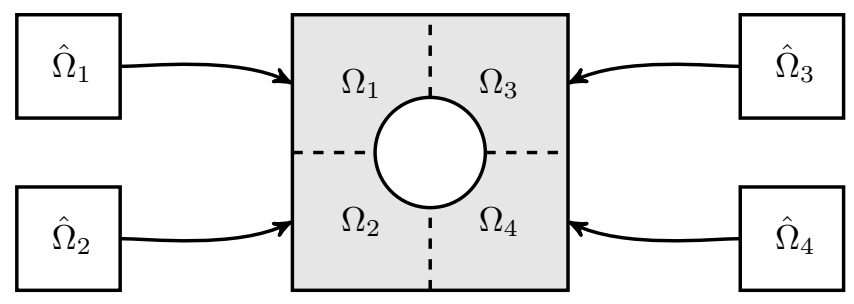

FIG. 2.3. Simple geometry that requires more than a single patch to be represented.

independent code. Then we give a detailed description of the main objects, generally accompanied with snippet of code to illustrate its use finishing with a simple code to assemble a local operator in Listing 13 that integrates some of the main classes.

3.1. Design overview. Following $\$ 2.1$, an IgM is a special case of a Galerkin method where the basis functions in the reference domain are of B-spline type (NURBS, T-spline, etc) and the basis functions in the physical domain are their push-forwards (see Table 2.1). The regularity of the mapping must be compatible with the one of the reference basis and the transformation type. In this conception of an isogeometric method the main mathematical concepts are the reference domain $\hat{\Omega}$, the reference space $\hat{\mathbb{V}}(\hat{\Omega})$, the physical domain $\Omega$, the mapping $\boldsymbol{F}: \hat{\Omega} \rightarrow \Omega$, the transformation type that together with the mapping gives the push-forward operator $P$ and the physical space $\mathbb{V}(\Omega)$. These concepts and their relations are depicted on the left column of Figure 3.1. In the physical space we are interested in handling quantities such as the differential operators $\mathcal{A}: \mathbb{V}(\Omega) \rightarrow \mathbb{V}(\Omega)^{\prime}$, source operators $f \in \mathbb{V}(\Omega)^{\prime}$, and fields $u \in \mathbb{V}(\Omega)$ (see lower part of Figure 3.1). We need access to these quantities both on the global level (where the system is solved and solution plotted) as well as on the local level (where the operators are assembled). Under the object oriented paradigm, a good software design for an IgM library is one that remains faithful to the mathematics of isogeometric analysis. The right column of Figure 3.1 sketches the design of igatools emphasizing the relation between the main concepts in an IgM (left column) in synchronization with the classes used to represent these concepts in the library.

3.2. Similarities and differences with the FEM. For the creation of our design it was important to identify the similarities and differences between the IgM and the FEM. One approach that has been proposed (10, 13]) to implement isogeometric codes is to add an isogeometric plugin on existing FEM software packages. This idea only exploits the similarities, and could make sense for specific engineering applications where decades of FEM coding has been invested. Another approach, which is the one we follow, is to take advantages not only of the similarities but also of the differences. Thus, we use the successfully proven finite element design ideas that also apply to an IgM but we do not include useless FE design ideas. Instead we create new concepts to produce a design naturally suitable for an IgM. The similarities come from both being Galerkin methods with basis functions of small support which makes convenient to perform the assembling by adding local contributions. Also the handling of the global system is similar but this is not even the ground of the FEM itself but of a linear algebra system. As far as the differences, we should start by saying that there are concepts in each of the methods that do not make sense for the other. For example in an IgM we have no master element, or finite element triple, or degrees of freedom associated with geometric objects of the triangulation, but we have concepts such as a reference space, a push-forward and a physical space, which on the ground of implementation have not made a lot of sense for a FEM code design. Having a design with native support for these concepts makes the whole approach to the IgM more natural and comprehensible, not only for a user but more so for new programmers that want to develop in the isogeometric concept without having to produce a forced translation to finite elements. Figure 3.2 pictorially compares the different concepts used in a FEM and an IgM to generate the physical space.

3.3. Dimension independent code. Besides the implementation of a new design, a very interesting feature of igatools is the capability of writing dimension and range independent code. All classes are 
Isogeometric Method Concepts

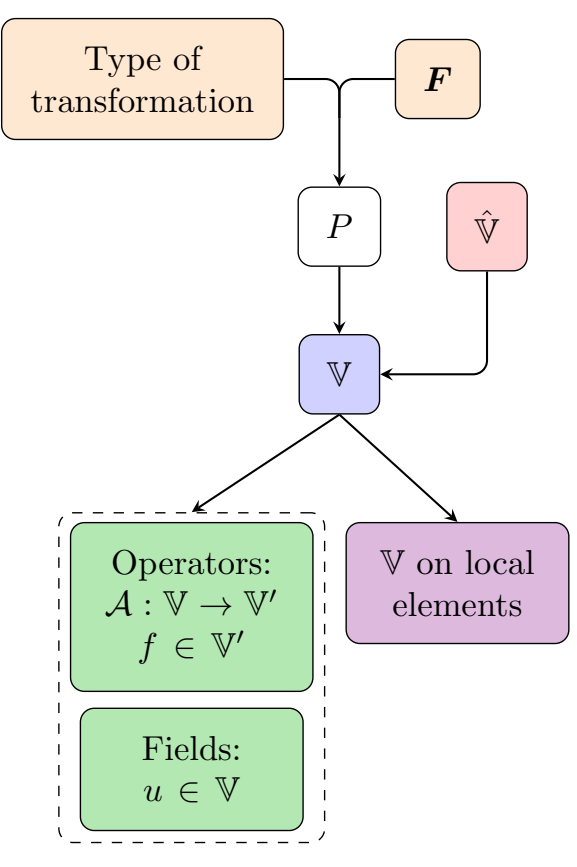

igatools Classes

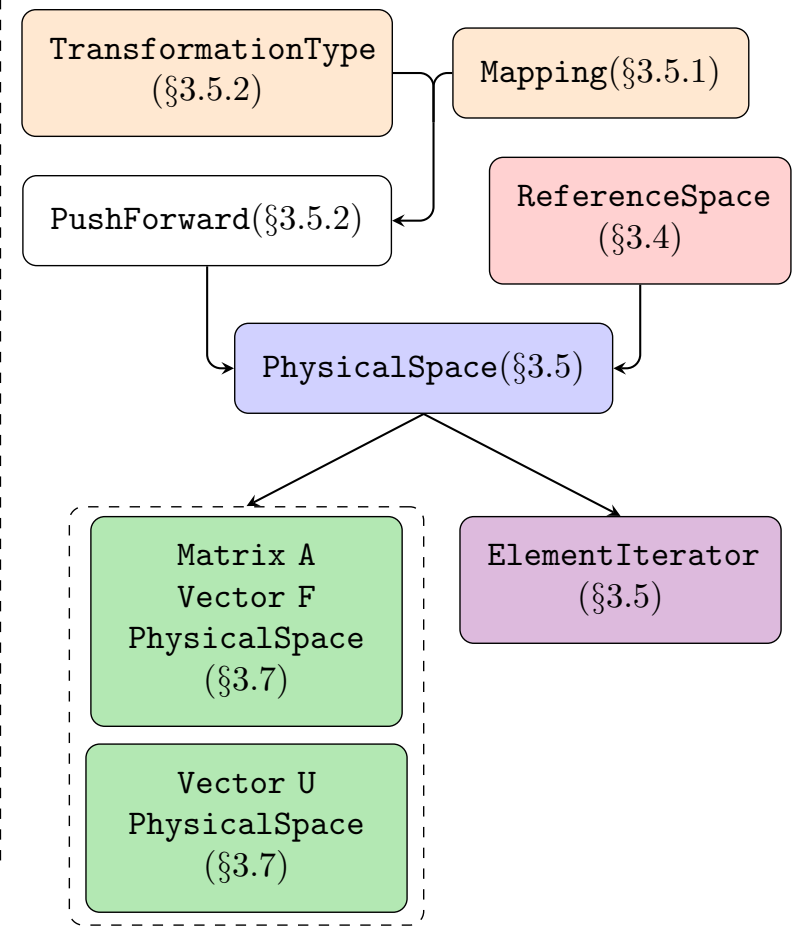

FIG. 3.1. The conceptual diagram sketches the IgM object oriented design advocated in this work and implemented in igatools, on the left column there are the mathematical concepts found in isogeometric analysis and on the right column the corresponding realization of such concepts through the main classes in igatools. Both location and color are used to emphasis the synchronization of concepts-classes and their interaction. Under the object oriented paradigm, a good software design for an IgM library is one that remains faithful to the mathematics of isogeometric analysis. The classes include a reference to the paper section where they are described.
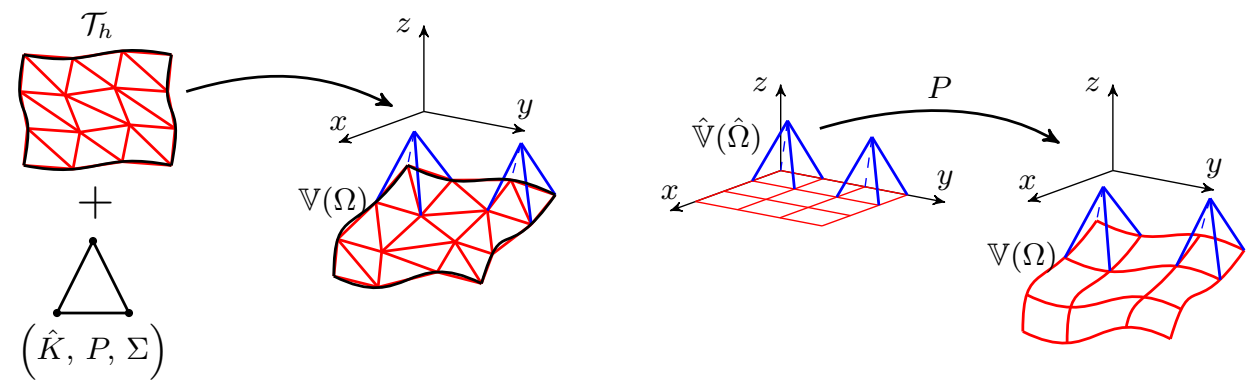

FIG. 3.2. Both the FEM and the IgM are particular cases of the Galerkin method. They provide a specific way of constructing a sequence of discrete approximating spaces. Both share the properties of having basis function with small support, but the way to generate the spaces is essentially different as illustrated in the picture. In the FEM, the approximating space $\mathbb{V}(\Omega)$ is constructed from the finite element triple $(\hat{K}, P, \Sigma)$ and the triangulation $\mathcal{T}_{h}$. In the IgM the physical space is generated from a global reference space and a push-forward operator.

designed in such a way that the space dimension, co-dimension and the tensorial range are selected as template parameters. The use of templates is very convenient for scientific computing, they allow to have a single code that is resolved at compile time generating optimized code as if it was written for each instance 
of the dimension and with no run-time checks that would affect performance. This approach allows us to write an application code that is independent of dimension and range (provided that the mathematical problem can so be formulated). For example, let's say that a user writes the code for a 2D problem that can be mathematically formulated in any dimension (for ex. Poisson's equation). If this code is written with some minimal care, then the same code will run for the problem with the physical domain being 1D, $3 \mathrm{D}$ or a 2D manifold embedded in $\mathbb{R}^{4}$ and for the solution being scalar-, vector- or tensor-valued (cf. 6.1 ). Table 3.1 shows an example of how the template technique for dimension independent code appears to the user in the case of the class BSplineSpace (3.4). The dimension independent approach can be discovered in any piece of code shown in this work.

\begin{tabular}{cc}
\hline$\hat{\mathbb{V}}(\hat{\Omega})$ is a spline space and $\hat{\phi} \in \hat{\mathbb{V}}(\hat{\Omega})$ & igatools realization of $\hat{\mathbb{V}}(\hat{\Omega})$ \\
\hline$\hat{\phi}: \mathbb{R}^{2} \rightarrow \mathbb{R}$ & BSplineSpace<2,1,1> v_hat $(\ldots) ;$ \\
$\hat{\phi}: \mathbb{R}^{3} \rightarrow \mathbb{R}^{3}$ & BSplineSpace<3,3,1> v_hat $(\ldots) ;$ \\
$\hat{\phi}: \mathbb{R}^{2} \rightarrow \mathbb{R}^{3 \times 3}$ & BSplineSpace<2,3,2> v_hat $(\ldots) ;$ \\
$\hat{\phi}: \mathbb{R}^{d} \rightarrow \mathcal{T}^{k}\left(\mathbb{R}^{s}\right)$ & BSplineSpace<d, s, k> v_hat $(\ldots) ;$ \\
\hline
\end{tabular}

TABLE 3.1

The library uses template parameters to attain dimension and range independent code, the table shows an example on how the code looks when constructing an object of the BSplineSpace type. The left column shows the domain and range of a spline function for a given spline space, and the right column the how igatools uses template parameters to define a spline space for that domain and range combination. The last row shows the generic format of this class. The second and third arguments can be omitted in which case the library assumes some default values.

3.4. Reference space (BSplineSpace and NURBSSpace). Currently the library provides two classes or types for the reference space $\hat{\mathbb{V}}(\hat{\Omega})$. These are BSplineSpace and NURBSSpace, realizations of the spaces $S_{\vec{\xi}}^{\boldsymbol{p}}(\hat{\Omega})$ and $N_{\overrightarrow{\boldsymbol{\xi}}}^{\boldsymbol{p}}(\hat{\Omega})$ described in $\$ 2.2$ (including their tensor-valued counterparts of $\$ 2.2 .4$. These classes can have different degrees and multiplicities in each coordinate direction and different scalar spaces for each range component. The last type of spaces are necessary for example in the context of fluid [25, 17]. We will use the generic class ReferenceSpace to refer to either BSplineSpace or NURBSSpace. In order to construct a reference space it is necessary to provide the reference domain $\hat{\Omega}$, the vector of knot vectors $\overrightarrow{\boldsymbol{\xi}}=\left(\boldsymbol{\xi}_{1}, \ldots \boldsymbol{\xi}_{d}\right)$, the degrees $\boldsymbol{p}=\left(p_{1}, \ldots p_{d}\right)$ and in the case of a NURBS space the weight function $\omega$. In igatools, the class CartesianGrid provides a realization for the vector of knot vectors without repetition as well as for the reference domain (a hyper-rectangle). Listing 1 shows example of creating CartesianGrids.

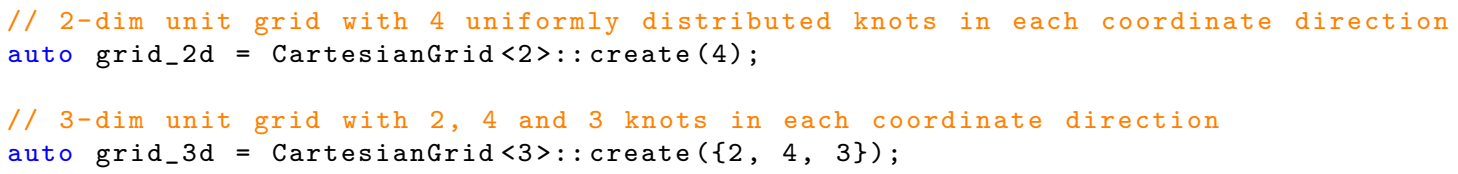

Creating CartesianGrids in igatools, some details are explained in the Listing as comments. The code also shows the use of the create idiom implemented in igatools for simple handling of smart pointers (cf. 4.3 ).

The vector with repetition $\overrightarrow{\boldsymbol{\xi}}$ is obtained from a CartesianGrid and a corresponding knot multiplicities (Multiplicity). The NURBS space weight function is passed through its coefficients. In Listing 2 we show some examples of constructing reference spaces.

3.5. Physical space (PhysicalSpace). The physical space $\mathbb{V}(\Omega)$ in igatools is realized by the class PhysicalSpace, this class needs to be provided with a RefereceSpace and a PushForward. The latter contains the geometry and transformation type. 


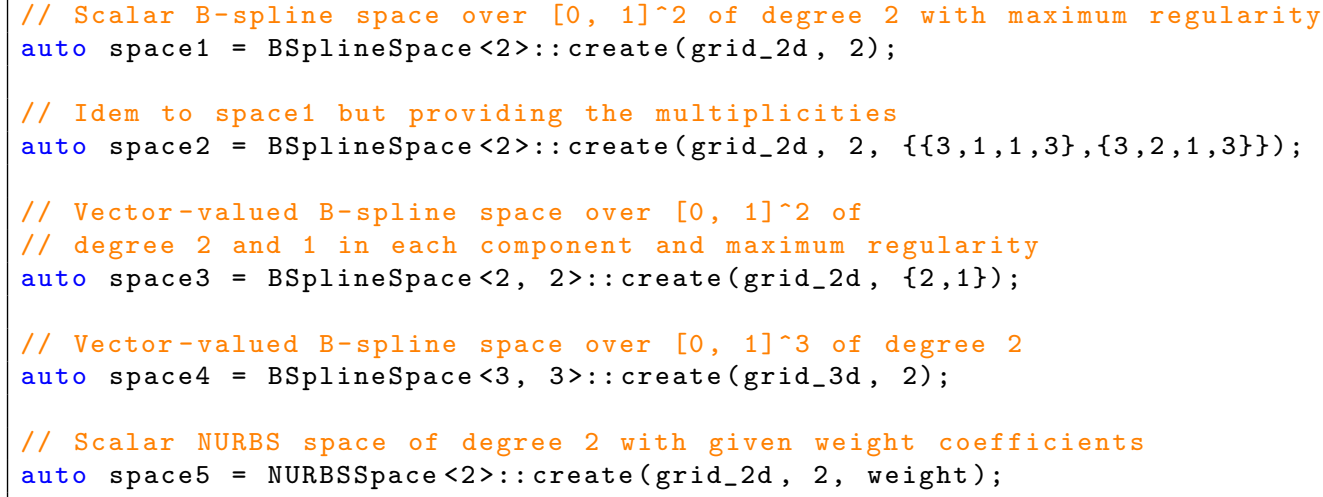

Creating reference spaces. Examples of constructing B-spline type spaces in igatools, some details are explained in the listing as comments. The code is referring to the grids created in Listing 1 .

3.5.1. Geometry (Mapping). The geometry or physical domain is described by the deformation $\boldsymbol{F}$ of the reference domain, in igatools the deformation is realized by the Mapping class. It is basically a function from $\mathbb{R}^{d}$ to $\mathbb{R}^{s}$ with the added feature of being element aware in the sense of $\$ 3.6$, so that its values can be handled with the grid-like iterators for uniformity and efficiency. Listing 3 shows examples of creating mappings. In particular, we remark that the encapsulation of the geometry in the Mappings

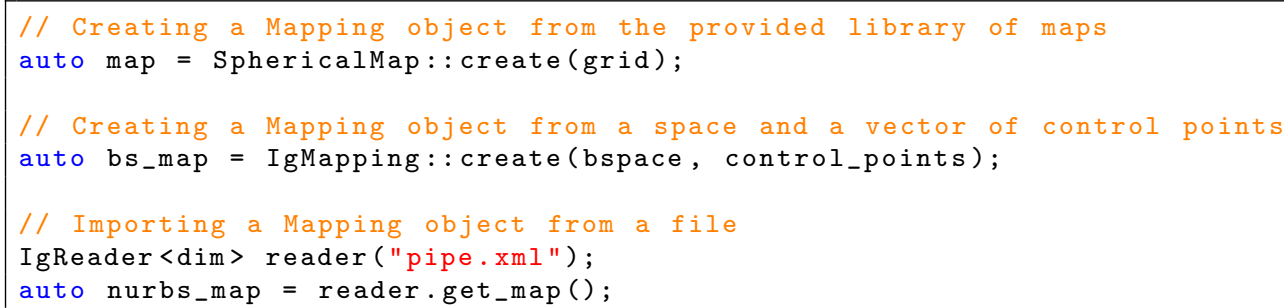

Code examples showing how to create some geometries (Mappings). The library allows the use of different types of deformations, including analytic functions and isogeometric mapping.

class allows to cleanly integrate a geometric modeler (or a CAD system) by implementing a Mapping specialization (see $\$ 5.2$ ).

3.5.2. Push-forward operator (PushForward). The other ingredient besides a reference space required to construct a physical space is a push-forward operator. In igatools it is realized by the PushForward class, which is constructed from a Mapping and a Transformation. Currently, igatools provides support for $\mathrm{h}_{-} g r a d, h_{-} d i v, h_{-}$curl and 12 transformation types (see Table 2.1). Listing 4 shows an example for creating a push-forward operator and a physical space.

3.6. Element level access (ElementIterator). The ReferenceSpace class can be thought of as a container of basis functions. The latter are supported only on a small number of elements, which makes it convenient to access them through the elements $\hat{Q}$ of the grid $\hat{\mathcal{Q}}$. We refer to it as the local or element level access to quantities and it is provided using an element iterator in the spirit of the standard template library (STL) [41. Simply speaking, given an object collection (the container), an iterator may be thought of as a type of pointer that has two primary operations: referencing one particular element in the container (called element access), and modifying itself so it points to the next element (called element traversal). The primary purpose of an iterator is to allow a user to process every element of a container 


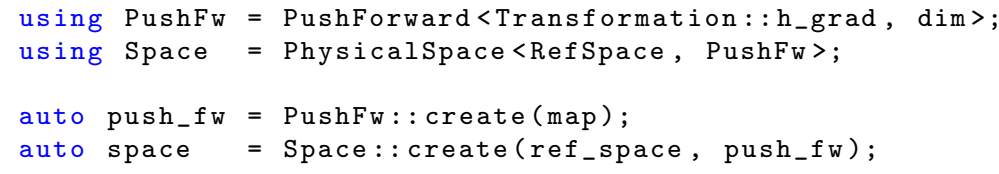

Creating a physical space. The physical space is created from a reference space and a push-forward operator. The push-forward operator is defined from the map using the $h_{-}$grad transformation type. The variables ref_space and map are assumed to have been defined as shown in Listings 2 and 3 respectively. Also notice the use of the using keyword to simplify long type names (see 4.3 ).

while isolating the user from the internal structure of the container. This allows the container to store elements in any manner it wishes while allowing the user to treat it as if it were a simple sequence or list. An iterator class is designed in tight coordination with the corresponding container class. In igatools we see the classes CartesianGrid, BSplineSpace, NURBSSpace, Mapping, PushForward and PhysicalSpace as containers of elements that we collectively call grid-like containers. The containers provide the methods for creating the iterators and thus a consistent way to iterate on the different data structures. In this way the code is more readable, reusable, and less sensitive to changes in the data structure (for example if we add support for local refinement). In particular, it fits perfectly in the dimension independent paradigm advocated by our design. Listing 5 shows a typical use of element iterators in igatools comparing the cases of a CartesianGrid, ReferenceSpace and PhysicalSpace. In particular notice the consistency in the treatment despite the different data stored in each class.

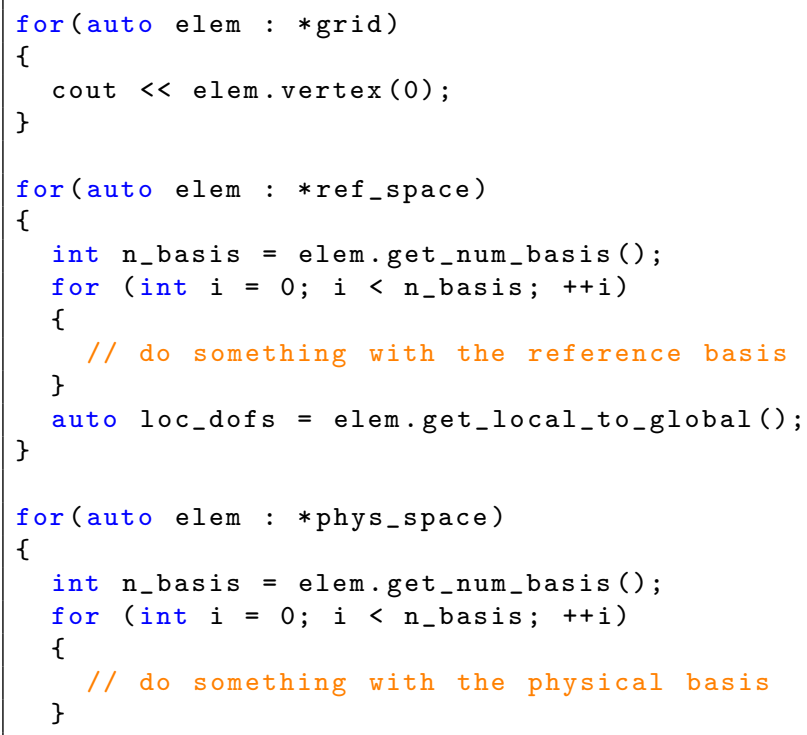

Uses of element iterators on igatools containers. We iterate on the elements of a CartesianGrid, a ReferenceSpace and a Physicalspace. Notice the consistent treatment that iterators allow to access different data. We assume that grid, ref_space and phys_space were defined as shown in listings 1 , 2 and 4 respectively.

A general purpose library like igatools cannot (and should not try to) guess what equation or problem the user will want to solve. Instead it should provide a flexible and intuitive way to access the quantities that seem to be common to all typical problems. Most operators require access to basis functions (values and derivatives of any order) and to geometric quantities (values and derivatives of the mapping, determinants and curvatures). Given the small support (in number of elements) of the basis functions, the assembling of 
the global quantities is conveniently performed by adding the local contributions. The ElementIterator is the mechanism that igatools provides to compute and access these values. In addition to the consistent interface to the different grid-like containers a second task that is handled by the element iterator is the management of an element values cache. A cache is a smart piece of memory that depending on the desired quantities to be computed stores commonly used computations to increase efficiency. Most optimization techniques tend to interfere with a clean code and it is common to have a trade off between efficiency and clean design. In order to partially counteract this effect we use the element iterator to implement an abstraction layer that is designed to be invisible from the neighbor layers. This provides the efficiency required but still keep an organized design for the caches. For using the cache mechanism the clean interface of the range based for loop shown in Listing 5 needs to be slightly modified. Listing 6 show the actual code for the assembling of the stiffness matrix (using the efficient cache mechanism) for a Poisson's problem. Obviously in the art of software design there is no technique to show that our design is the right

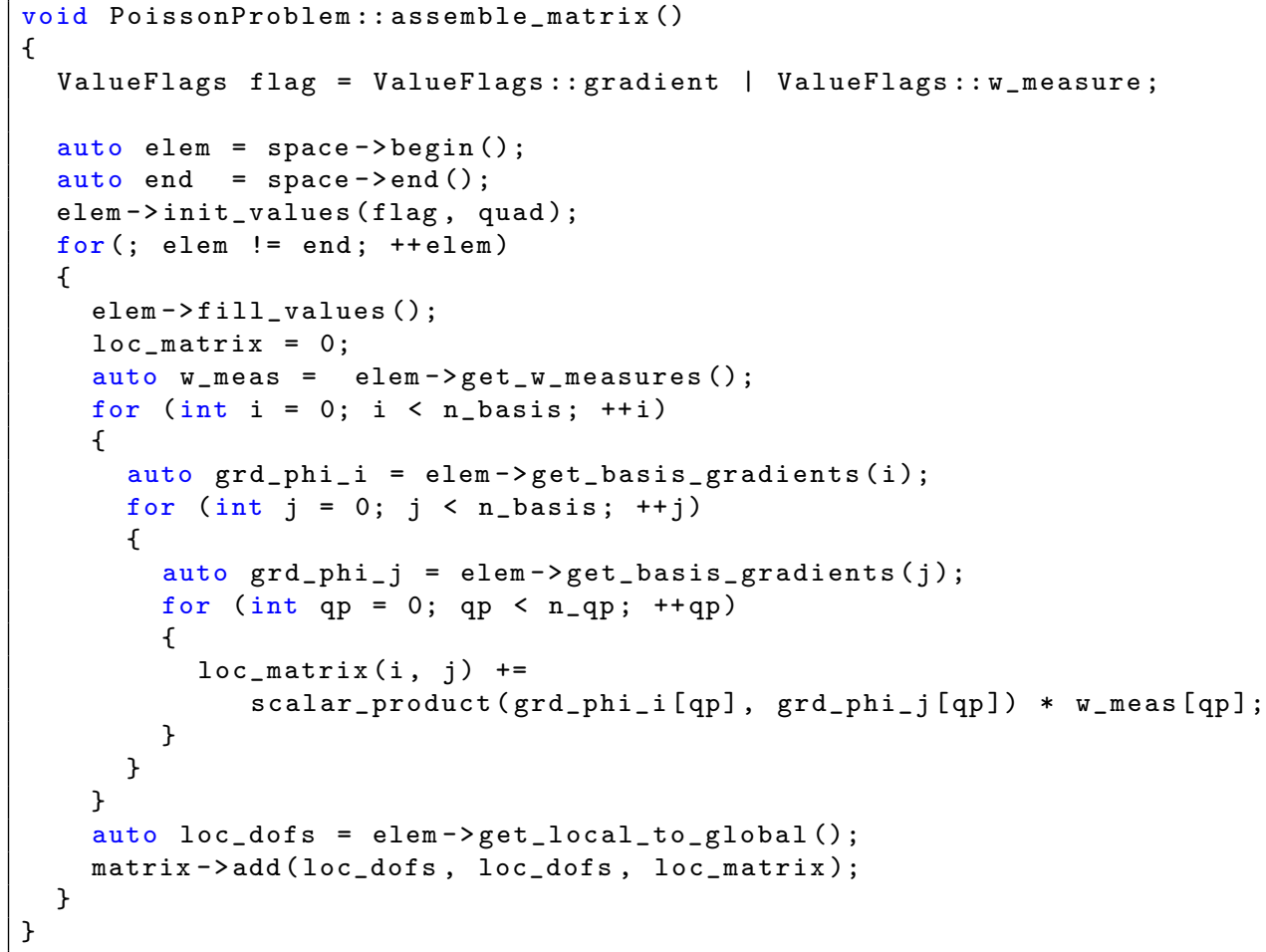

Code for the assembling of the stiffness matrix for the Poisson problem (cf. \$6.1. In order to use the efficient cache mechanism the range based for loops of Listing 5 needs to be modified, by adding the lines [5, 6. 7 and 10] Notice that init_values() is execute one time and fill_values() for each element.

one, but we find encouraging that the assembly function looks simple and plain enough also to non-coding experts, without sacrificing efficiency.

3.7. Global quantities: fields, operators and the linear algebra. We have discussed the local handling of basis functions to assemble the local contribution of operators. The global quantities, such as fields and global operators, are stored in containers of the linear algebra system (e.g. global vectors and matrices). They are assembled from the local element contributions through the bookkeeping mechanism kept by the space. For each element, the non-zero basis functions have a local index for which space knows the corresponding global index. The query for this information is obtained through the element iterator through the get_local_to_global () function Listing 6, line 26). Almost any of the many excellent linear 
algebra systems could be easily plugged into the proposed design. From a bias preference of the original developers (and for the sake of selecting one), in igatools we have used the linear (and non-linear) algebra provided by some packages of the Trilinos library [29. Trilinos, among other things, provides state of the art storage for distributed vectors and matrices as well as a complete collection of linear and non linear solvers. In order to have a consistent style with the other pieces of the library, igatools provides simplified wrappers to basic Trilinos vectors, matrices and linear solvers. At the same time we do not restrict the more advanced and powerful use that can be obtained by dealing directly with the Trilinos object.

4. igatools implementation standards. The effort in igatools has been directed to develop a high quality implementation of the isogeometric software design presented in $\$ 3$. To partially justify the statement, in this section we briefly describe some of the software engineering tools and development model we have adopted.

4.1. Development infrastructure. The development of igatools is supported by today's standard sofware engineering tools for that purpose. We use the distributed version control system and source code management git ${ }^{2}$, and the bug tracking system Trad 3 . For managing the build process, we use CMake ${ }^{4}$ allowing the software compilation process using a simple platform and compiler independent configuration files. Most importantly, we strive for high standards for documentation and user support. The documentation can be divided in three level: this paper, that describes the design concepts and how they work together; the tutorial examples provided with the source to give a hand-on introduction to use the library; and a reference manual generated by processing the in-source documentation with Doxygen ${ }^{5}$. For community support we have a user groun ${ }^{6}$ for discussion between the igatools developers and users and a development groun 7 where the igatools developers discuss about new features, library design, implentation details, discovered bugs and possible remedies. We also mantain a wiki page 8 working as the webpage to the world of the library.

4.2. Development model (testing and debugging). igatools has hundreds of unit tests (small programs) that are run automatically using CDash/CTest $\mathrm{S}^{9}$ to verify that new changes are not breaking working features.

We use and advocate the use of the test driven development model [8]. This basically means that we write a unit test for the feature we want to implement or fix, the test initially fails, then we write code until the test passes, from that moment on the test is executed automatically. Now we can refactor the code (clean, remove any duplication, rename variables and methods, optimize, etc.) being confident that the new code is not damaging any existing functionality. In addition to the unit tests, igatools has automatic integration and validation tests (more cpu-time expensive than unit tests) in order to verify that the different modules interact correctly.

The libray makes use defensive programming techniques to detect and easily find bugs at runtime, through the exception handling mechanism. We adopt two levels of checks. One, more expensive in terms of running time, but only active when igatools is compiled in Debug mode. We perform this kind of checks wherever there may be a chance of error, e.g. out-of-bound index for accessing to vectors elements, uninitialized objects, invalid object states, mismatching dimensions, etc. The second level of checks is always active (both in Debug and in Release mode) and it is used for checking anomalies that may be introduced by the input data.

The typical workflow for a user of igatools would be to first write his code and test it with the library

\footnotetext{
2 http://www.git-scm.com

3 http://trac.edgewall.org

${ }^{4}$ http://www.cmake.org

5 http://www.doxygen.org

6 https://groups.google.com/forum/\#!forum/igatools-users

7 https://groups.google.com/forum/\#!forum/igatools-development

$\varepsilon$ http://code.google.com/p/igatools/w/list

http://www.cdash.org
} 
in Debug mode on a small-size problem. When this is working as expected and (virtually) bug-free, link the code with igatools in Release mode on a real-size problem.

4.3. Programming language. The main language used in the development of igatools is $\mathrm{C}++1132$. It includes many additions to the previous standard C++03 31, mostly aimed to produce a code that is cleaner, safer, more efficient and easier to maintain. In particular, igatools makes heavy use of some of the language features somehow defining certain aspects of its programming style, as for example:

(i) Smart pointers and create facility. Objects in igatools can have big sizes and can be shared by different other objects. This sharing mechanism is handled through the use of smart pointers. They provide automatic memory management ensuring that the resource they control is automatically destroyed when its last (or only) owner is destroyed. Moreover, the igatools classes intended to be used through smart pointers provide a create facility for this purpose. For each constructor of the class there is a static function (called create) with the same arguments of the constructor that returns an instance of the class wrapped by a shared pointer. A code example showing the implementation and use of the technique is shown in Listing 7 .

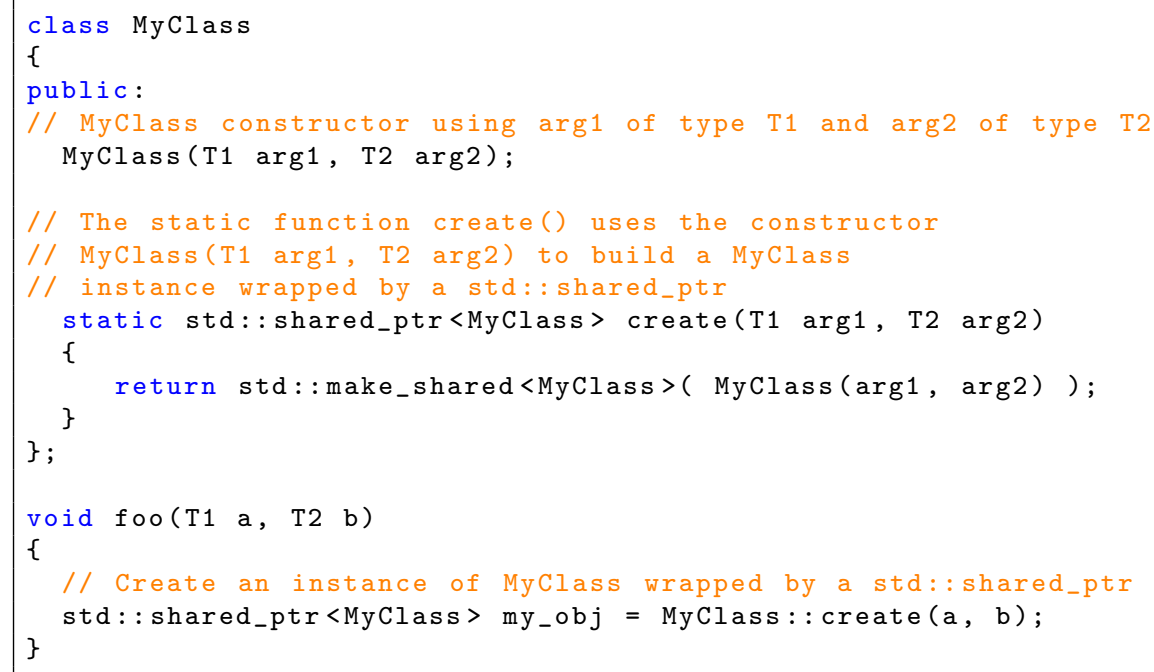

Create facility. For each class that is intended to be used through a smart pointer, igatools provides a create facility. The static function create returns an instance of the class wrapped by a std:shared_ptr. The function foo shows how the pointer is created.

(ii) Template types (using and auto). $\mathrm{C}++11$ provides better management of templates than $\mathrm{C}++03$ and igatools extensively uses templates in order to attain efficiency and the dimension independent paradigm. One drawback of using templates is the fact that the template arguments may become inhumanly difficult to express. We solve this problem with the $\mathrm{C}++11$ using alias facility, that allows to create human readable aliases for the necessary types. Related is the automatic type inference facility auto that deduces the type of an explicit initialization. An appropriate combination of using, create and auto results in a much more simple and human comprehensible syntax, as shown in Listing 8 , creating a certain style to code with igatools.

5. igatools practical features. Besides implementing the novel object oriented design presented in this work with high quality standards, igatools provides useful features that make it useful and attractive for practical applications.

5.1. Input/output facilities. It is important for the library to use NURBS geometries generated by other software. At the present stage there is no scientific computing dedicated format for isogeometric type of spaces. The developers of igatools and GeoPDEs in a joint effort defined an xml format to describe such 


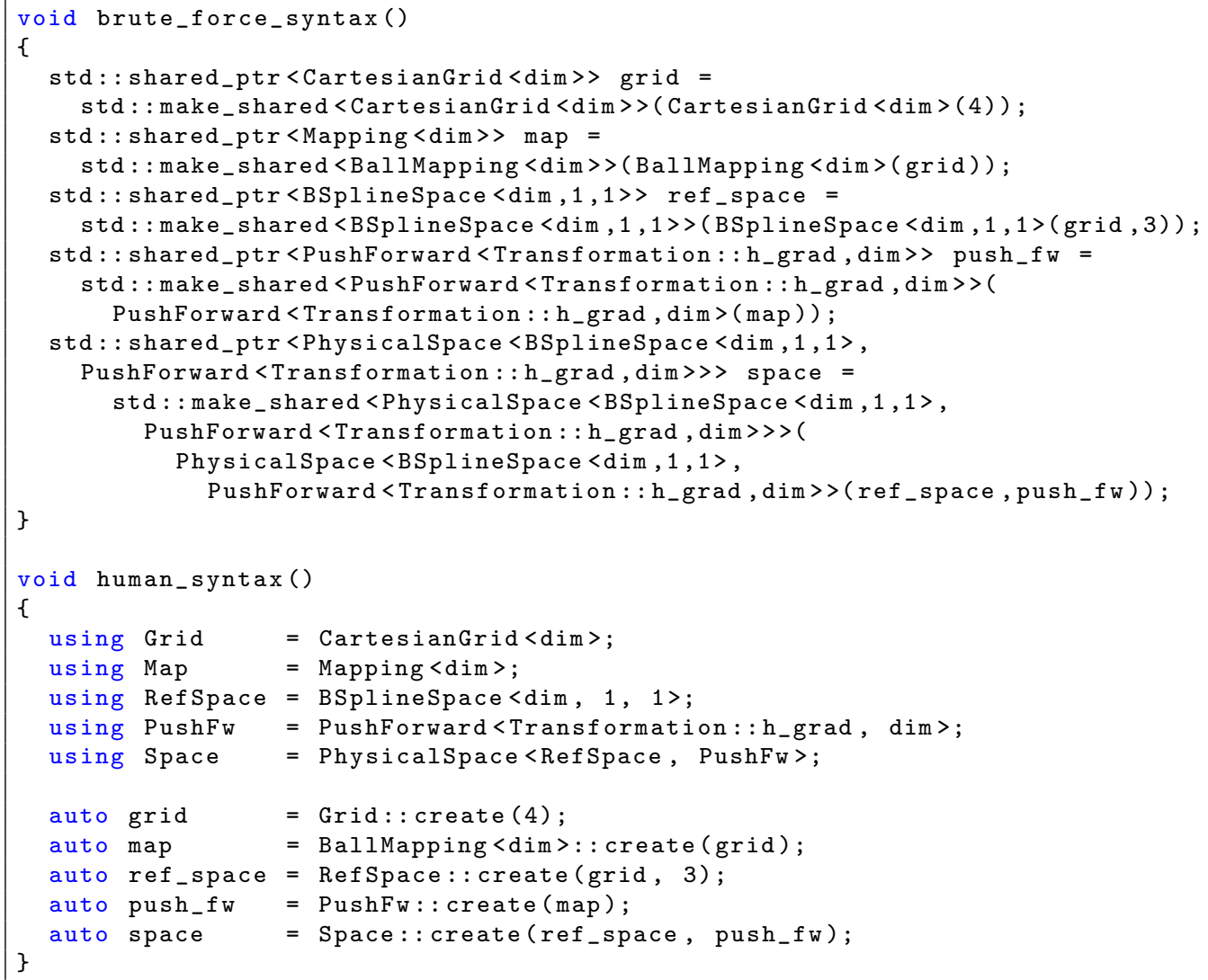

Library facilities using, auto and create. The two functions perform the same operations, but the second one uses the combination of the using, auto and create facilities advocated by the igatools programming style, resulting in a more human readable syntax than the brute force counterpart.

geometries. Thus igatools, through it IgReader class (see Listing 3 , lines 8 and 9 supports importing geometries in this data format, which is completely specified in the library documentation. xml is an extremely flexible format that is supported by wide number of libraries and applications. igatools source is distributed with contributed scripts to convert geometries generated with the MATLAB NURBS toolbox 10 to the xml format.

Similarly important for the library is to provide a useful mechanism to analise and visualize the computational results. igatools generates output for this purpose through its Writer class (see Listing 15 ). So far the writer provides output in vtk format 37] which can be visually processed (among other things) with Paraview 40 .

5.2. Interaction with CAGD software. The main selling point when isogeometric analysis was introduced was the possibility to integrate the geometry with the analysis. In the context of igatools this can be cleanly achieved by implementing a Mapping specialization (see 3.5 .1 that internally use all geometric features of a modern CAD system, provides a clear interface to the user without him worrying about the implementation details. Now, after a decade from the introduction of IGA there is no dedicated CAD software that directly generates a 3D analysis suitable geometry, this being one of the limiting issues in the use of the IgM in the industrial community. One reason is that most CAD software when dealing with a 3D geometries, only model the boundary surfaces of the represented domain. An exception is the

10 http://www.aria.uklinux.net/nurbs .php3 
geometric modeler IRIT ${ }^{11}$ developed by G. Elber at Technion, that natively handles trivariate volumes. We have tested the integration of igatools with this particular geometric modeler, by implementing a Mapping specialization (MappingIRIT) that internally uses the IRIT's routines and data structures for the evaluation of the geometric quantities required by Mapping. This MappingIRIT class is used for the geometry in the non-linear elasticity example presented in 6.4

5.3. Parallel support. Computationally demanding applications require igatools to take advantage of parallel computing technologies. The Trilinos and Intel TBB ${ }^{12}$ libraries, used in cooperation with igatools, provides this service using a distributed and shared memory model respectively. The containeriterator model separates data from operations. In fact the iterators are designed in such a way they do not modify their container. In this way we can obtain parallel iterators, where each thread points to different grid elements. We have successfully tested the use of Intel TBB in igatools, following very closely the implementation of the deal. II classes for the threads management. In particular a correct management of threads/iterators for the parallel assembly routines has been checked. A distributed memory model using MPI have been succesfully applied to igatools application code by using the Trilinos facilities for a distributed assembling and parallel solving of the global system.

6. Examples. In this section we illustrate the library flexibility to naturally handle and adapt to different situations. We do this by showing its use to solve typical application problems. We start with a rather detailed description for the implementation of a simple Poisson equation. This is the de facto prototype example in any Galerkin numerical method that we also employ to describe the basic building blocks typical to the use of the library and to which we refer to in the subsequent examples. We continue with a tiny adaptation to the Poisson problem code that solves the Laplace-Beltrami problem on a manifold. Then we illustrate the handling of vector-valued and many spaces problems by discussing the implementation of the Stokes equation. Finally, we present a non-linear elasticity problem showing the flexibility of the library to cleanly interact with other software packages.

Notice that in the snippets of code listed in the section some non-relevant pieces are omitted (or more precisely replaced by a comment of the form "//...") to help with the understanding of the main pieces. Also as we move on in the examples, less details are provided as we assume they can be translated from the previous examples.

6.1. Poisson Equation. The Poisson problem consists in finding $u: \Omega \subset \mathbb{R}^{d} \rightarrow \mathbb{R}$ such that

$$
-\Delta u=f \text { in } \Omega, \quad u=g \text { on } \partial \Omega .
$$

It can be rewritten in weak form as: find $u=u_{0}+u_{g}$, with $u_{0} \in H_{0}^{1}(\Omega)$ and $u_{g}$ the lift of $g$, such that $\mathcal{A} u(v)=F(v)$ for all $v \in H_{0}^{1}(\Omega)$, where $\mathcal{A} u(v)=\int_{\Omega} \nabla u \cdot \nabla v$ and $F(v)=\int_{\Omega} f v$. The IgM for this problem (cf. \$2.1) requires: the definition of the isogeometric space $\mathbb{V}(\Omega)$; the assembling of the stiffness matrix $\boldsymbol{K}$ and right hand side $\boldsymbol{F}$ by adding the element contributions; and the solving of the linear system $\boldsymbol{K} \boldsymbol{U}=\boldsymbol{F}$.

Below we describe a possible approach for the implementation of this problem with igatools. The full running code for this problem is part of the tutorial distributed with the library. To be concrete, we choose: the physical domain $\Omega$ to be a $d$-dimensional spherical sector for $d=1,2$ and 3 ; a homogeneous boundary condition; and a constant source of 5 . The code would look practically the same if we decide to use a CAD geometry for the domain, a non-homogeneous boundary function and/or a non-constant source term.

In order to undertake this problem, a class for it (see Listing 9p is defined, its public interface provides two members, the constructor PoissonProblem and the run() function. Within the private members one can identify the declaration of objects for the space and the linear algebra. We can also see the using keyword to create short alias for the usually long templated types and the use of smart pointers (shared_ptr), both common techniques within the programming style of igatools (cf. 4.3 ). For the actual solving of the problem we define objects of PoissonProblem type in the program's main() (see

\footnotetext{
11 http://www.cs.technion.ac.il/ irit/

12 https://ww. threadingbuildingblocks.org/
} 


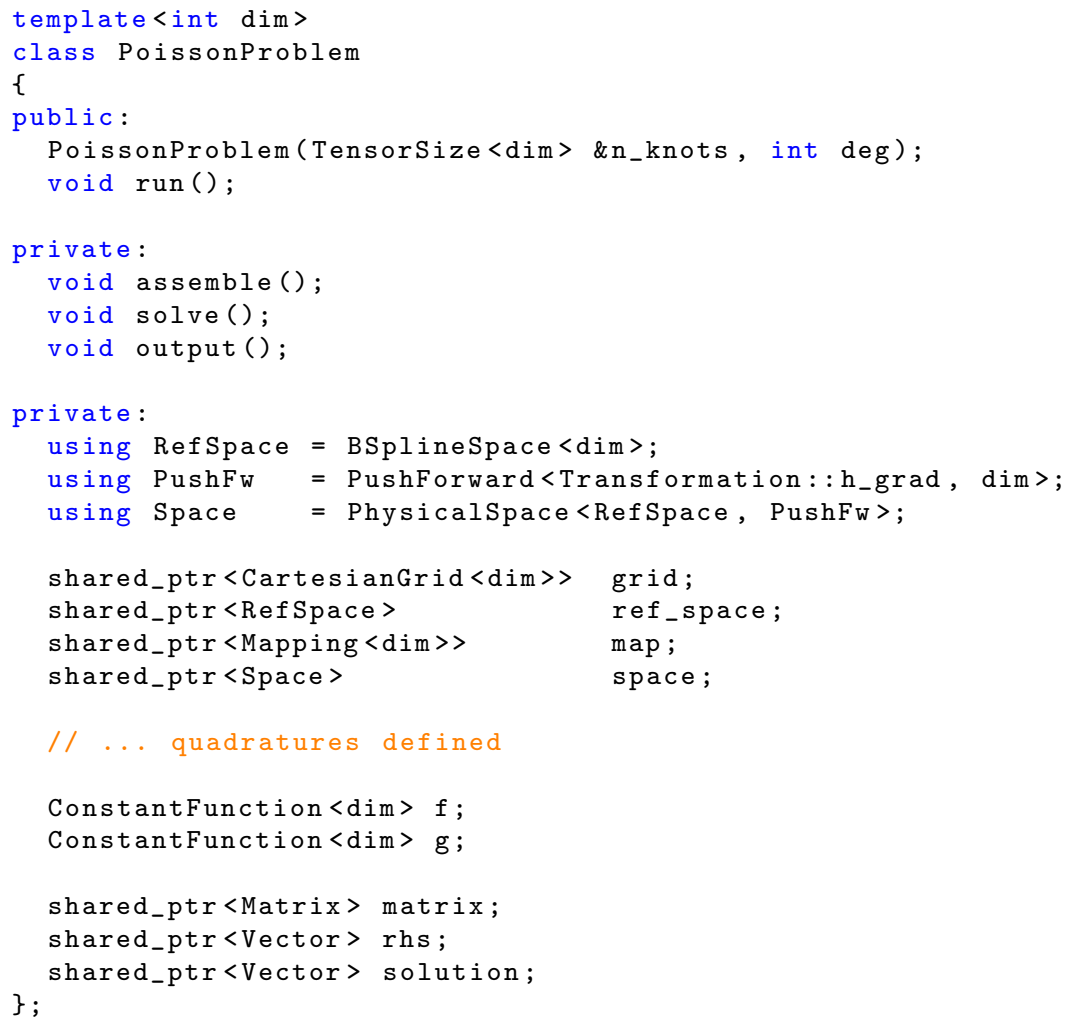

Class to approximate the solution of a Poisson problem. The public interface provides the constructor that prepares the object and the function run() that computes and plots the solution. In the private members we can find objects to handle the space and the linear algebra.

Listing 10p. Here we can see how the dimension independent template technique (cf. 3.3 ) is put to

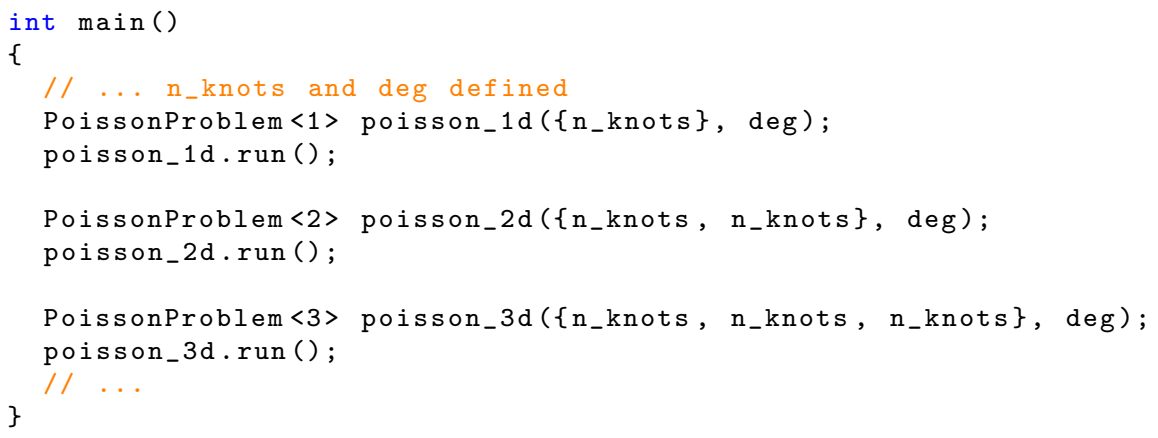

Main routine to solve the Poisson problem for different domain dimensions. For each dimension 1,2 and 3 we construct the problem and then assemble, solve and output the result by calling the function run().

use. We are solving the Poisson problem in 1,2 and 3 dimensions with the same templated code. As a matter of fact, with the same code we could solve this problem in 4 dimensions if we wish to. Each object definition (lines 4, 7 and 10 calls the constructor that prepares the object to be ready to use and then we call the object member function run() that computes the solution. The function run() (see Listing 


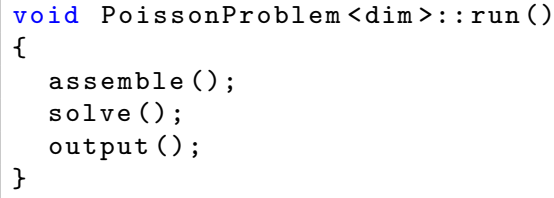

The run function is the public interface that solves the Poisson problem by assembling and solving the discrete linear system and saving the solution in graphical format.

11) is just a high level interface that calls the private members assemble(), solve() and output () that will do the actual computational work and are explained later on. After executing this program, graphical output files (in vtk format) for the different dimensions with the problem solution are saved. Figure 6.1 shows these files.
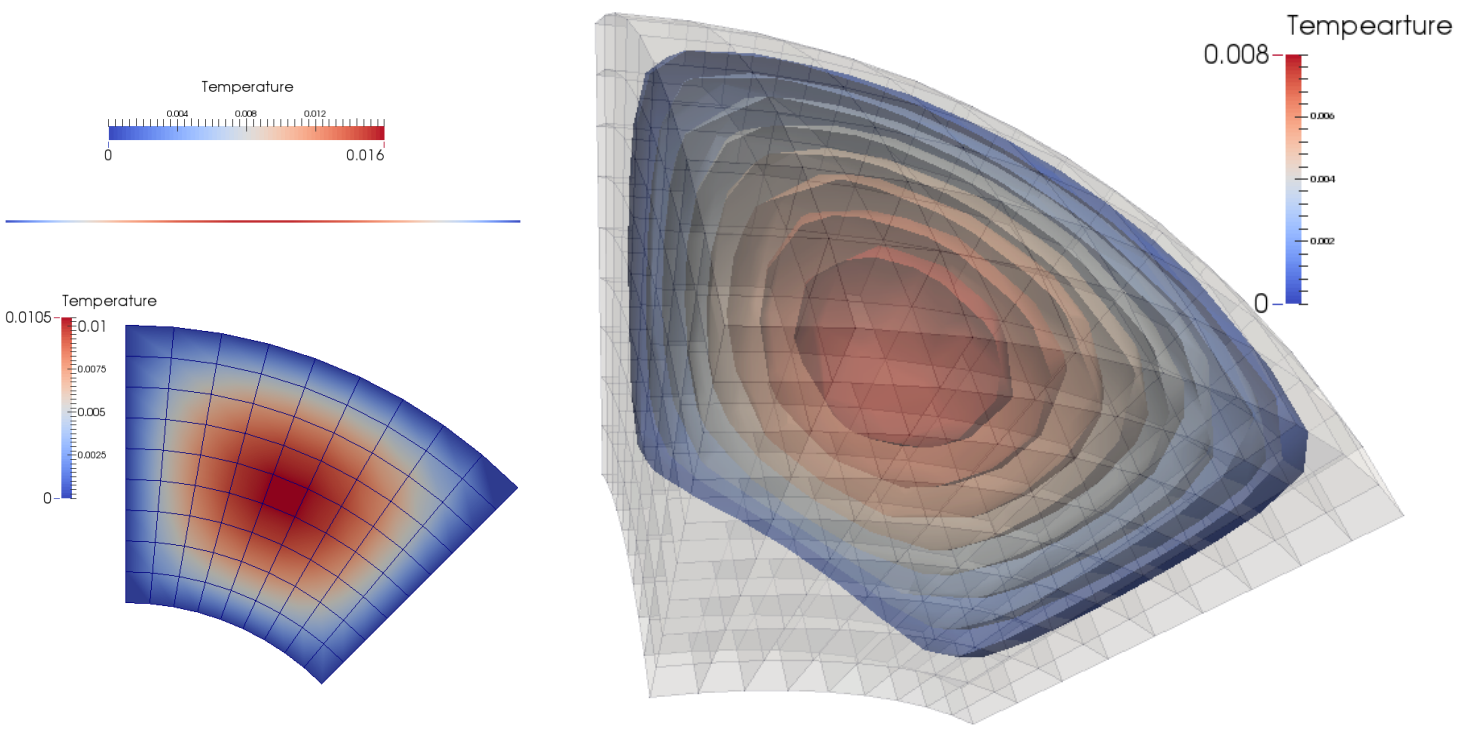

FIG. 6.1. Plot of the solutions to the Poisson problem with homogeneous boundary conditions and constant source term on a spherical sector in 1, 2 and 3 dimensions. Notice that the three plots were generate with the same code, by only changing the dimension template argument.

Now we explain the implementation of the different member functions of the PoissonProblem class. The constructor (see Listing 12 is in charge of making the object ready to be used. So it creates a physical space space by first creating a grid, a reference space ref_space, a mapping map and a push forward.

The assemble() routine assembles the global stiffness matrix matrix and global right hand side rhs by adding the element contributions. In igatools we use the element iterator (\$3.6) for this purpose. We have already seen in Listing 6 the assemble of the global matrix, here in Listing 13 we include the assembling of the right hand side and the treatment of the boundary conditions. In this case we proceed in two steps (lines 27 and 28), first we project the boundary function $g$ onto the trace space and then we enforce into the linear system these values for the Dirichlet degrees of freedom. Notice that a projection is necessary as splines are not interpolatory in general.

As discussed in 3.7 , igatools relies on external algebra packages for solving the global system. The function solve() in Listing 14 defines a Solver object which is a wrapper class provided by igatools for basic use of Trilinos solvers. The solution of the system is returned in solution. The lines in this listing look simple enough to be self-explanatory.

To manage the graphical output ( $\$ 5.1$, igatools provides a Writer class that can be used to handle 


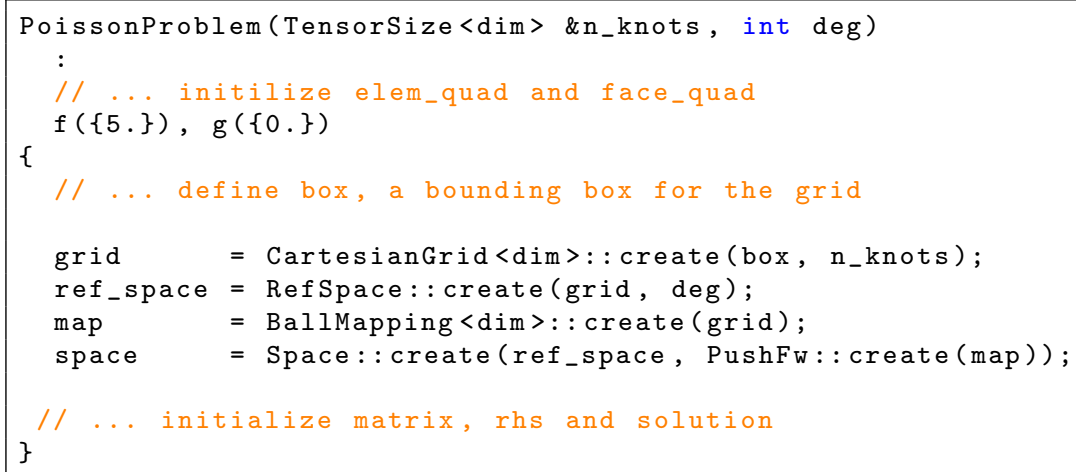

LISTING 12

Poisson's problem constructor. It construct the physical space, for which it needs to construct a reference space and a push-forward which in turn requires the construction of a map and a cartesian grid. It also initializes the quadrature schemes, the linear algebra and the source and boundary functions.

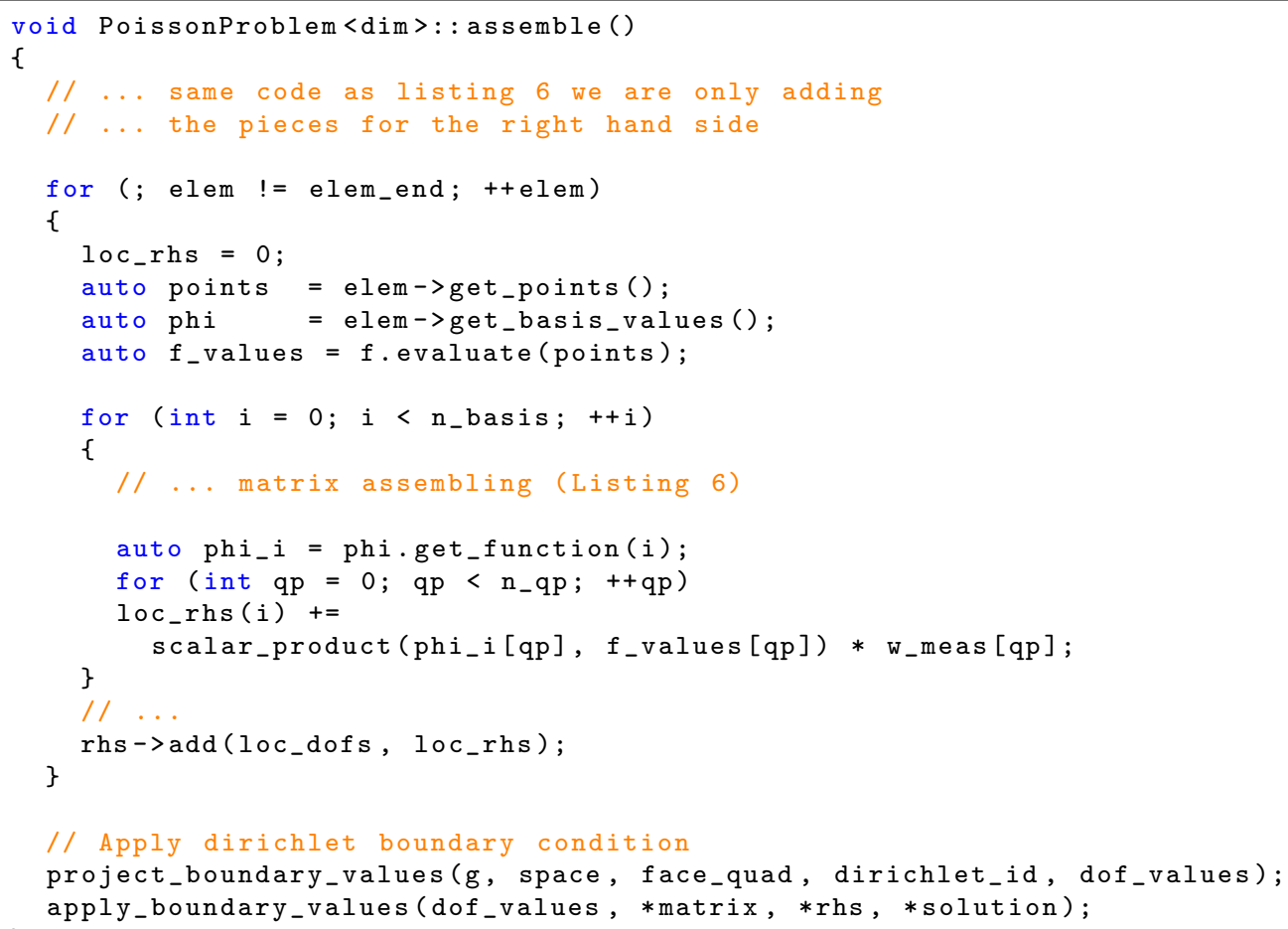

Assembling of the global system for Poisson problem. The matrix assembling was already shown in Listing 6 , here we only show the additional code required to assemble the right hand side and take care of the dirichlet boundary constraints.

geometries and fields that are to be saved in a graphical output format (for now a vtk file). In Listing 15 a Writer object associated with the geometry of map is defined (line 4), then the solution field is added to it (line 5), and finally writer is asked to save the geometry and the solution field to disk (line 6).

6.2. A surface example. The code of 6.1 can be trivially modified to solve a partial differential equation on a surface. igatools can naturally handle manifold domains such as surfaces or curves.

Let $\Gamma$ be a $d$-dimensional manifold, and consider the Laplace-Beltrami equation: find $u: \Gamma \rightarrow \mathbb{R}$ such 


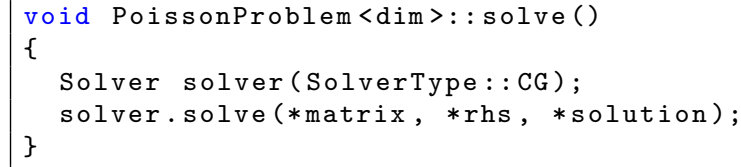

Solver for the Poisson problem. We use the simple wrapper igatools provides to the Trilinos solvers.

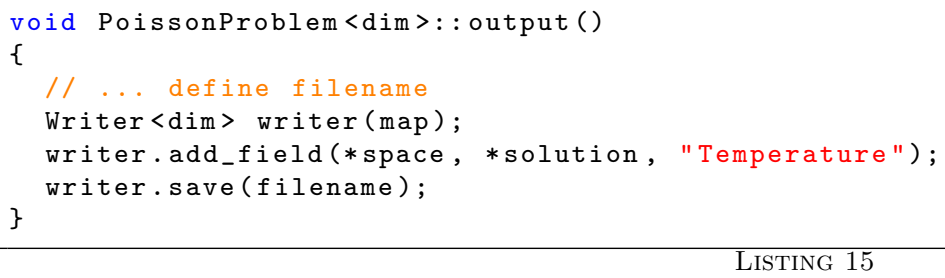

Saving the solution of Poisson problem in a graphical output.

that

$$
-\Delta_{\Gamma} u=f \text { in } \Gamma, \quad u=g \text { on } \partial \Gamma,
$$

which can be rewritten in weak form as find $u=u_{0}+u_{g}$, with $u_{0} \in H_{0}^{1}(\Gamma)$ and $u_{g}$ the lift of $g$, such that $\mathcal{A} u(v)=F(v)$ for all $v \in H_{0}^{1}(\Gamma)$, where $\mathcal{A} u(v)=\int_{\Gamma} \nabla_{\Gamma} u \cdot \nabla_{\Gamma} v$ and $F(v)=\int_{\Gamma} f v$. Except for the fact that the differential operators are surface operators, one can see the similarity of this problem with equation 6.1). The library understands manifolds, thus when the physical space has codimension different from 0 , igatools understand that gradients refers to surface gradients. In this way, minor modifications to the Poisson problem code of 6.1 allow to solve the Laplace-Beltrami problem.

More precisely, without loss of generality, let assume we want to solve equation (6.2) on a $d$-dimensional spherical piece of codimension 1 . It only requires minor changes on a few lines in the problem class, first the definitions of codim and space

static const int codim $=1$;

static const int spacedim = dim + codim;

and then some small changes in lines 15, 20, 25 and 26 of Listing 9 by the following four lines in the given order

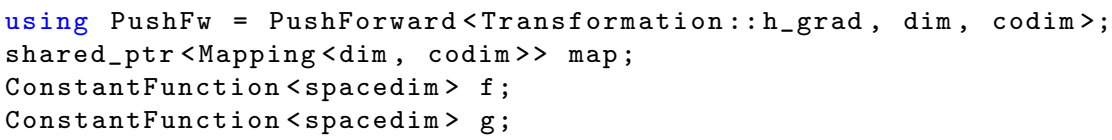

The only change required in the constructor is to replace line 10 of Listing 12 by

map $=$ SphereMapping $\langle$ dim $>:$ : create (grid);

In Figure 6.2 we plot the isogeometic approximate solution to the Laplace-Beltrami problem over a spherical piece given by a spherical coordinate map.

6.3. Navier-Stokes Equations. An incompressible viscous fluid flowing in with a laminar motion is modeled by the Navier-Stokes equations

$$
\rho\left(\partial_{t} \mathbf{u}+(\nabla \mathbf{u}) \mathbf{u}\right)-\mu \Delta \mathbf{u}+\nabla p=\mathbf{f} \quad \text { and } \quad \operatorname{div} \mathbf{u}=0 \quad \text { in } \Omega \times(0, T),
$$

supplied with appropriate initial and boundary conditions. Here $\mathbf{u}$ is the fluid velocity field and $p$ its pressure. A distinctive attribute of these equations is the combination of hyperbolic and parabolic terms 28. The key to the application of the Galerkin method to the weak form of these equations lays into 


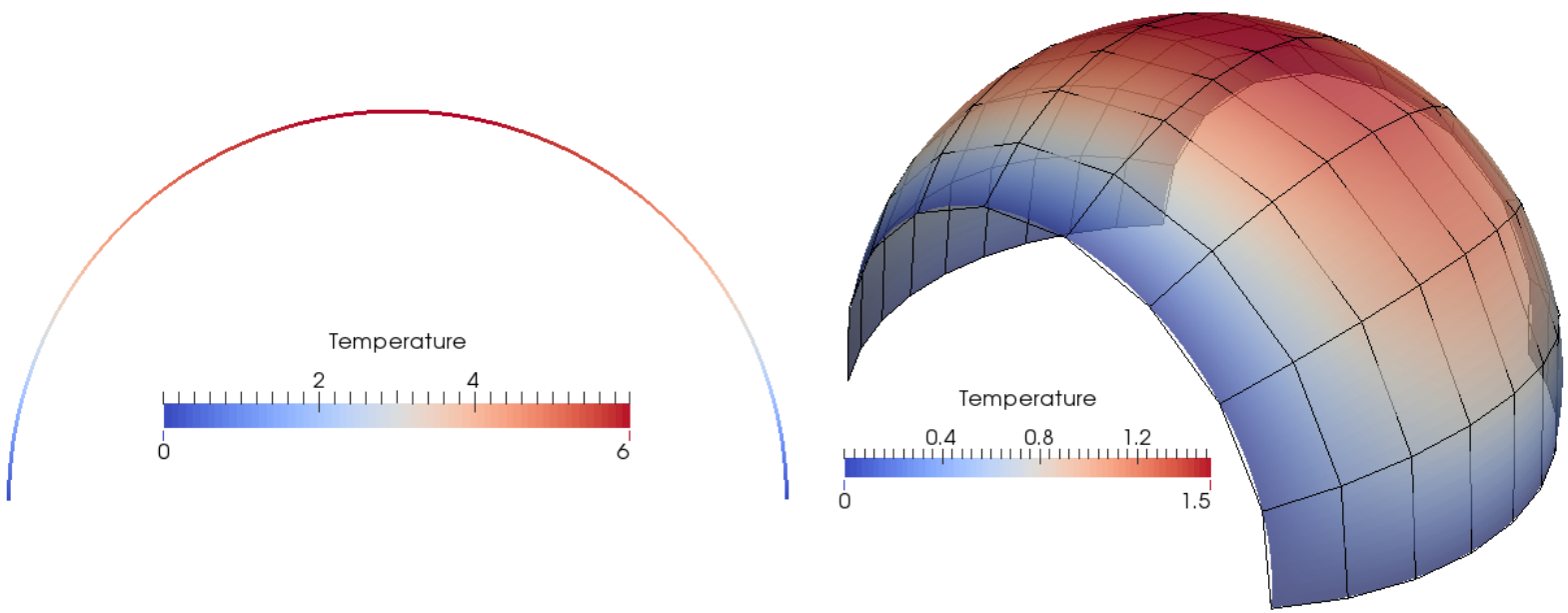

FIG. 6.2. Plot of the solution to the Laplace-Beltrami problem with homogenous boundary condition and constant source term on a piece of a d-sphere, for $d=1,2$.

its parabolic term with the incompressibility constraint leading to a saddle point problem. In particular, the spaces for velocity and pressure have to satisfy the well-known inf-sup condition [11. We assume that $\mathbb{V} \subset H^{1}(\Omega)^{d}$ and $\mathbb{Q} \subset L^{2}(\Omega)$ is a pair of spaces for the velocity and pressure that satisfy this condition, exactly what subspaces are taken depends on the type of boundary conditions. The discrete problem is obtained by considering discrete spaces $\mathbb{V}_{h} \subset \mathbb{V}$ and $\mathbb{Q}_{h} \subset \mathbb{Q}$ for the velocity and pressure respectively, and can be written in matrix form as:

$$
\left(\begin{array}{cc}
\boldsymbol{A} & \boldsymbol{B}^{t} \\
\boldsymbol{B} & \mathbf{0}
\end{array}\right)\left(\begin{array}{l}
\boldsymbol{U} \\
\boldsymbol{P}
\end{array}\right)=\left(\begin{array}{c}
\boldsymbol{F} \\
\mathbf{0}
\end{array}\right)
$$

where the submatrix $\boldsymbol{A}$ corresponds to the terms involving the fluid velocity and $\boldsymbol{B}$ corresponds to the mixed term. For the discretization of this problem we use two spaces: one scalar-valued (for the pressure) and the other vector-valued (for the velocity). Most importantly, they should form a stable pair (i.e. satisfy a discrete inf-sup condition).

Below we illustrate the use of igatools to treat the two coupled spaces by listing the relevant pieces of code for a Stokes type of problem. Listing 16 shows part of the StokesProblem class declaration. In particular, notice the scalar-valued space type PreSpace being similar to the space of section 6.1 while for the VelSpace type the addition of the second template parameter renders it into a vector-valued space.

For the purpose of this example we consider the Taylor-Hood type of spaces adapted to the isogeometric setting [4, 14. In which the pressure and velocity spaces share the same global regularity and the velocity degree is one more that of the pressure. These spaces are constructed in the StokesProblem constructor (see Listing 17). Here, given the degree for the pressure space and the global regularity as arguments, the constructor builds the multiplicity vectors pre_mult and vel_mult and the degrees pre_deg and vel_deg for both spaces, which are created in lines 17 and 18 . Recall that to have a global regularity $r$ in a spline space of degree $d$ the multiplicity of the interior knots must be $d-r$ and the end knots to be interpolatory require a multiplicity $d+1$.

An interaction between the velocity and pressure spaces is required, for example, in the assembling of the mixed term $\boldsymbol{B}^{t}$. This involves the simultaneous access to basis functions from both spaces over the same element and quadrature points. Listing 18 illustrates how this is attained in igatools using two element iterators. We use the iterators pre_el and vel_el, one for the pressure and one for the velocity space in a common for loop, with a simultaneous increment. This works because both spaces are defined on the same CartesianGrid, so the increment operator ensures that both iterators traverse the elements in synchronization. 


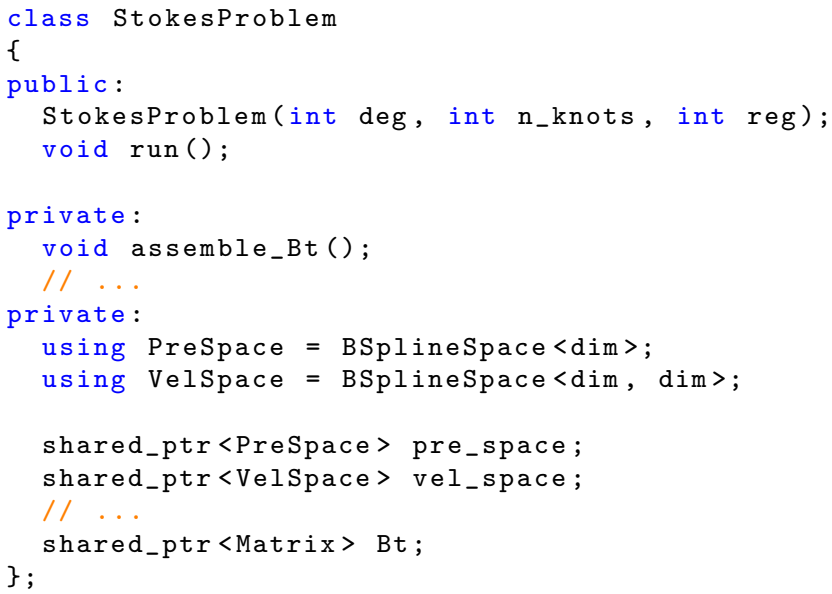

LISTING 16

Sketch of a class to solve the Stokes problem. Observe the declaration of two spaces one vector-valued for the velocity and one scalar-valued for the pressure.

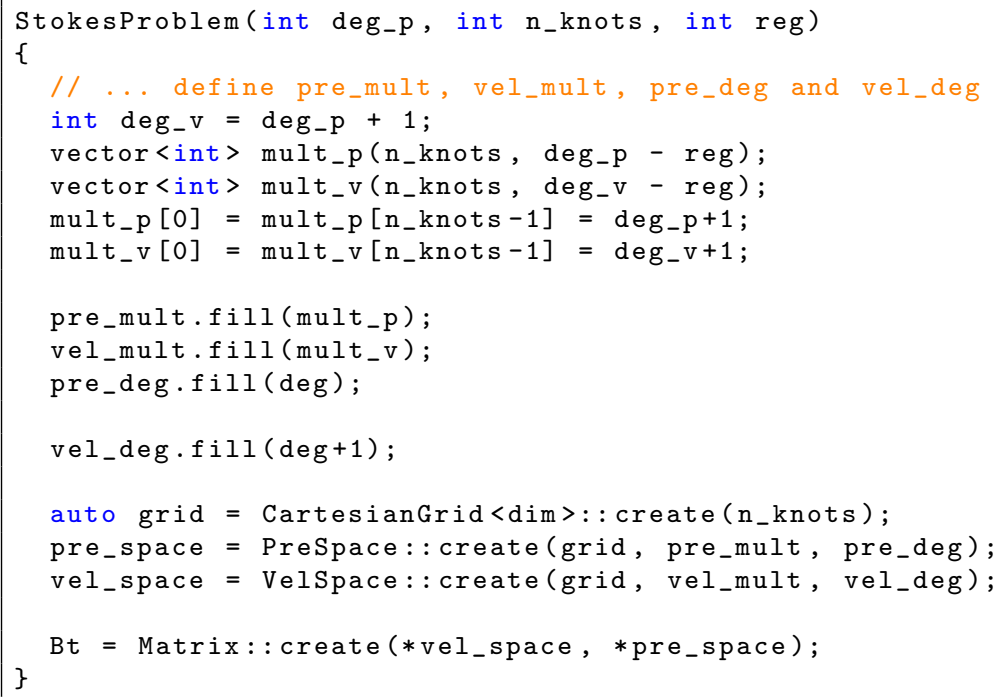

LISTING 17

Constructor for Stokes class. The scalar and vector-valued spaces for the isogeometric version of the Taylor-Hood elements are created. The pressure and velocity spaces share the same global regularity and the velocity degree is one more that of the pressure.

In Figure 6.4 we show a simulation run with igatools to solve a time dependent Navier-Stokes equations on a domain given by a quintic B-spline map. The time integration is performed using a first order operator splitting technique, see 28 as a reference. The geometry is sketched in Figure 6.3, it is inspired in vascular valves leaflets but there is no claim to model anything. Our main motivation is using our software in a non-trivial geometry. The geometry size is a 1 by 5 box. The leaflet tip is modeled with three control points separated by a distance of $10^{-3}$. The geometry mapping as well as the Taylor-Hood spaces have a $C^{4}$ global regularity, with degree 5 and 6 for the pressure and velocity respectively. Figure 6.4 shows the resulting pressure and streamlines at different time frames. Boundary conditions are no-slip at the bottom side, unitary horizontal velocity at the inlet, symmetry on top, and no stress at the outlet. The 


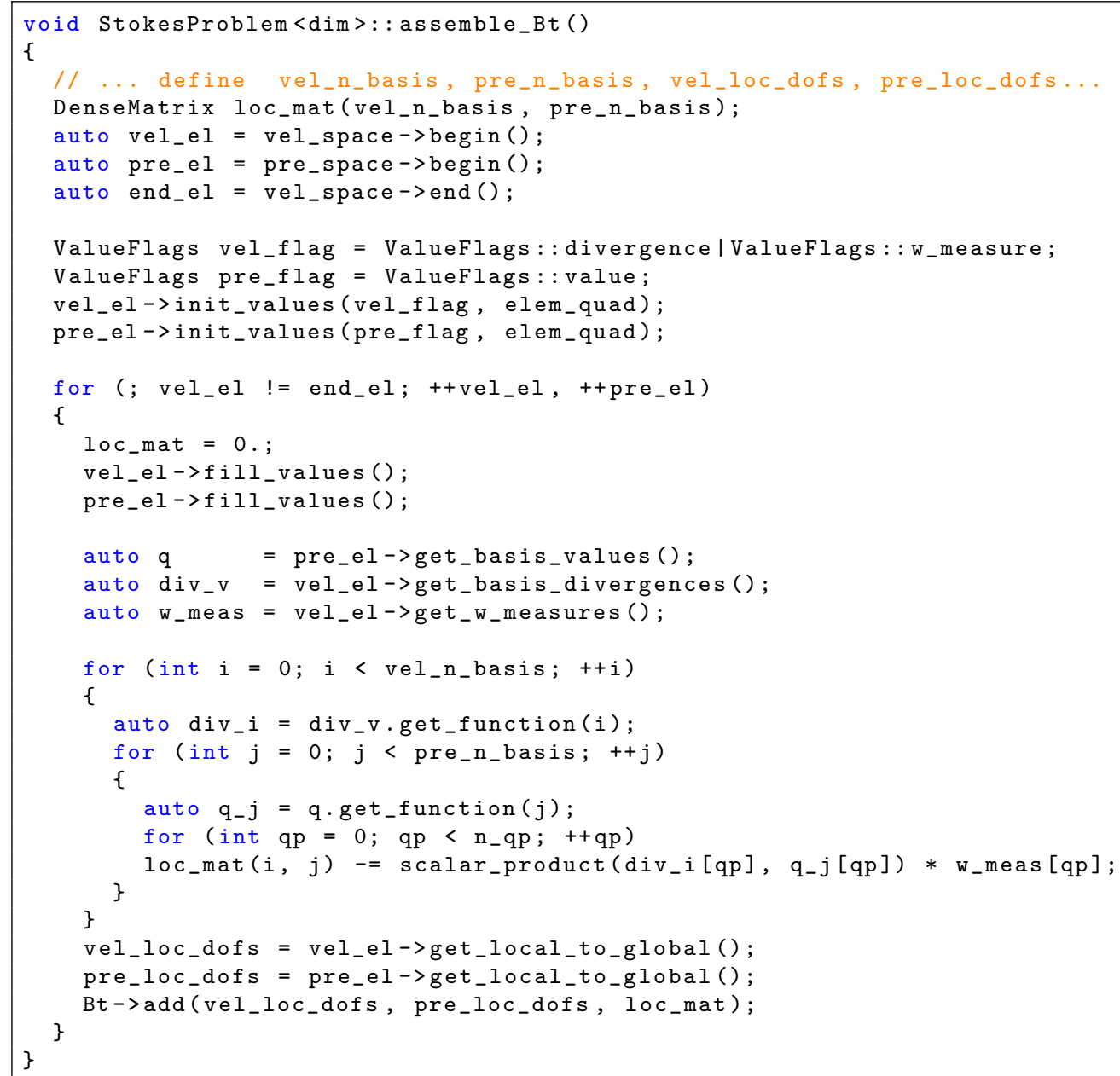

Assembling $B^{t}$ involves simultaneous access to the basis functions of both the pressure and velocity spaces. igatools approach is the use of two element iterators that are incremented simultaneously.

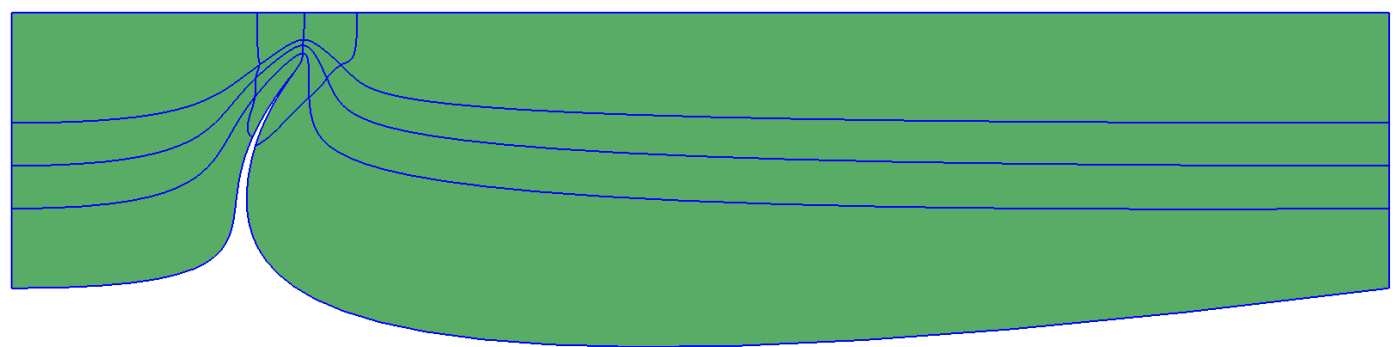

FIG. 6.3. Domain given by a quintic spline deformation of the unit square inspired in a vascular leaflet.

main vortex originates, as expected, from the leaflet tip. As time evolves, a secondary vortex originates from the bottom of the leaflet. 


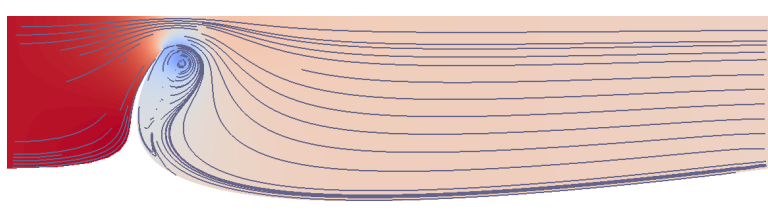

(a) $t=0.1$

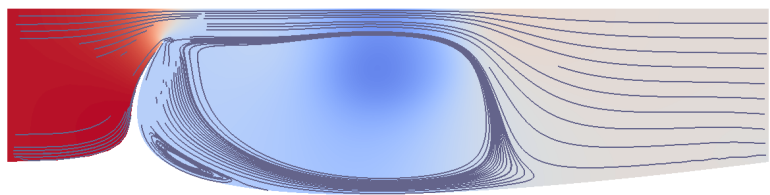

(c) $t=0.9$

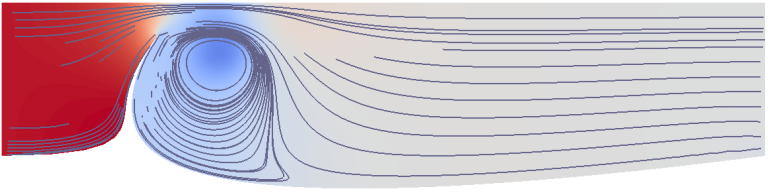

(b) $t=0.3$

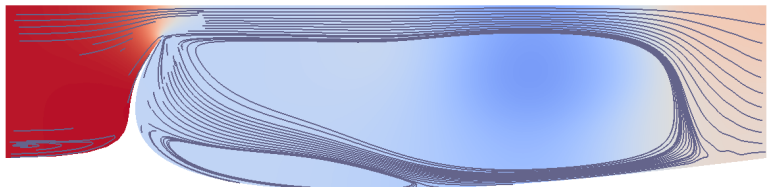

(d) $t=1.5$

FIG. 6.4. A time dependent Navier-Stokes simulation for the rigid leaflet geometry of Figure6.3. For illustrative reasons we decided to reproduce the shape of a vascular leaflet without any particular modelling motivation, we were interested in the vortex shedding by this geometry. In particular we notice the main vortex started at the tip of the leaflet, and a second one downstream at the bottom of the leaflet. The colormap represents the pressure.

6.4. Non-linear elasticity. The library design provides a flexible access to the isogeometric spaces. This flexibility and encapsulation permit easy integration not only with different algebra systems but also with other scientific packages for specific purposes. This section presents an example in which we have integrated igatools with FEBio [34, a non-linear finite element software for biomechanics.

The behavior of an elastic body is characterized by the equations

$$
\operatorname{div} \boldsymbol{\sigma}+\boldsymbol{f}=\mathbf{0} \text { in } \Omega ; \quad \boldsymbol{\sigma} \boldsymbol{n}=\boldsymbol{t} \text { on } \Gamma_{\sigma} ; \quad \boldsymbol{u}=\overline{\boldsymbol{u}} \text { on } \Gamma_{u},
$$

where $\boldsymbol{\sigma}$ is the Cauchy stress tensor; $\boldsymbol{f}$ and $\boldsymbol{u}$ are the body force and displacement vectors, respectively; $\boldsymbol{n}$ is the outter surface normal; and $\boldsymbol{t}$ is the surface traction vector. For this example we assume a non-linear constitutive relation between $\boldsymbol{\sigma}$ and $\boldsymbol{u}$ that leads to a non-linear problem.

The variational form of the equation $(6.4)$ can be written as

$$
\delta W(\varphi, \delta \boldsymbol{v})=\int_{\Omega} \boldsymbol{\sigma}: \nabla \delta \boldsymbol{v} \mathrm{d} \Omega-\int_{\Omega} \boldsymbol{f} \cdot \delta \boldsymbol{v} \mathrm{d} \Omega-\int_{\Gamma_{\sigma}} \boldsymbol{t} \cdot \delta \boldsymbol{v} \mathrm{d} \Gamma=0,
$$

being $\varphi$ the map such that the current (deformed) configuration is expressed as $\Omega=\varphi(\hat{\Omega})$. At the discrete level this equation can be expressed in a vector form as $\delta W(\varphi, \delta \boldsymbol{v})=\delta \mathbf{v}^{\mathrm{T}} \mathbf{R}=0$, where $\mathbf{R}$ is the residual vector. Due to the fact that $\mathbf{R}$ depends non-linearly on the unknowns vector $\mathbf{u}$ it is needed to perform a linearization of this equation. The matrix form of the linearization is $\mathbf{K}\left(\varphi_{k}\right) \Delta \mathbf{u}_{k}=-\mathbf{R}\left(\varphi_{k}\right)$, being $\mathbf{K}$ the stiffness matrix (further details can be found in [12]). They are referred to as the deformed configuration of the body $\Omega_{k}=\varphi_{k}(\hat{\Omega})$. An iterative procedure in $k$ is applied for achieving an equilibrium configuration $\varphi_{e}$ such as $\mathbf{R}\left(\varphi_{e}\right) \approx \mathbf{0}$, for a given tolerance.

igatools has been integrated with FEBio in a minimally invasive way: only the input/output and the computation of the element right-hand side vector and stiffness matrix have been modified, meanwhile linear algebra, degrees of freedom, material level computations, etc., are still managed by FEBio. The IGAElasticDomain class (Listing 19 materializes this integration, being derived from the FEBio's class FEBioElasticDomain it adds the isogeometric components such as the physical space. As it can be seen, a NURBSpace is used for the displacement. The final integration occurs in the functions for assembling the global stiffness matrix $\mathbf{K}$ and residual vector $\mathbf{R}$ by iterating over the elements and computing local contributions. For example, Listing 20 shows the assembling of $\mathbf{K}$ by computing the local matrices $\mathbf{K}^{e}$ given by

$$
\mathbf{K}_{i, j}^{e}=\int_{\Omega_{e}} \nabla B_{i}: \mathcal{C}: \nabla B_{j} \mathrm{~d} \Omega+\int_{\Omega_{e}} \nabla B_{i} \cdot \boldsymbol{\sigma} \nabla B_{j} \mathrm{~d} \Omega
$$




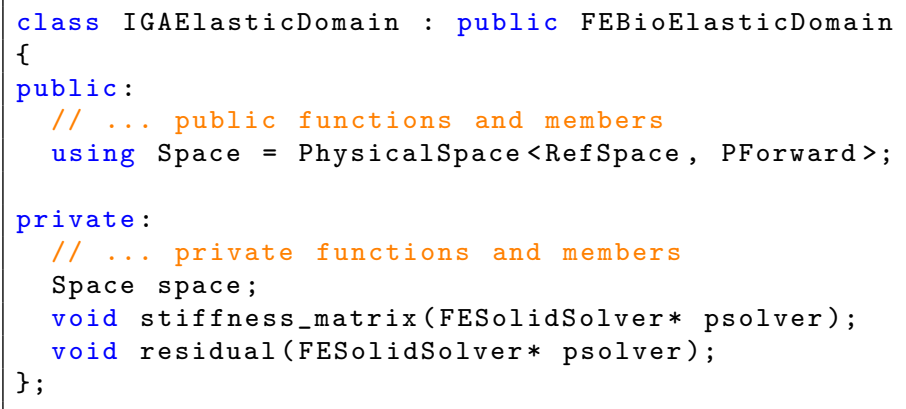

\section{LISTING 19}

Class for the solution of the non-linear elascity problem by means of the IgM integrated into FEBio. The spaces and push forward types are shown, as well as the public functions for assemblying the residual vector and the stiffness matrix.

to be then added, in line 29, into a global FEBio matrix.

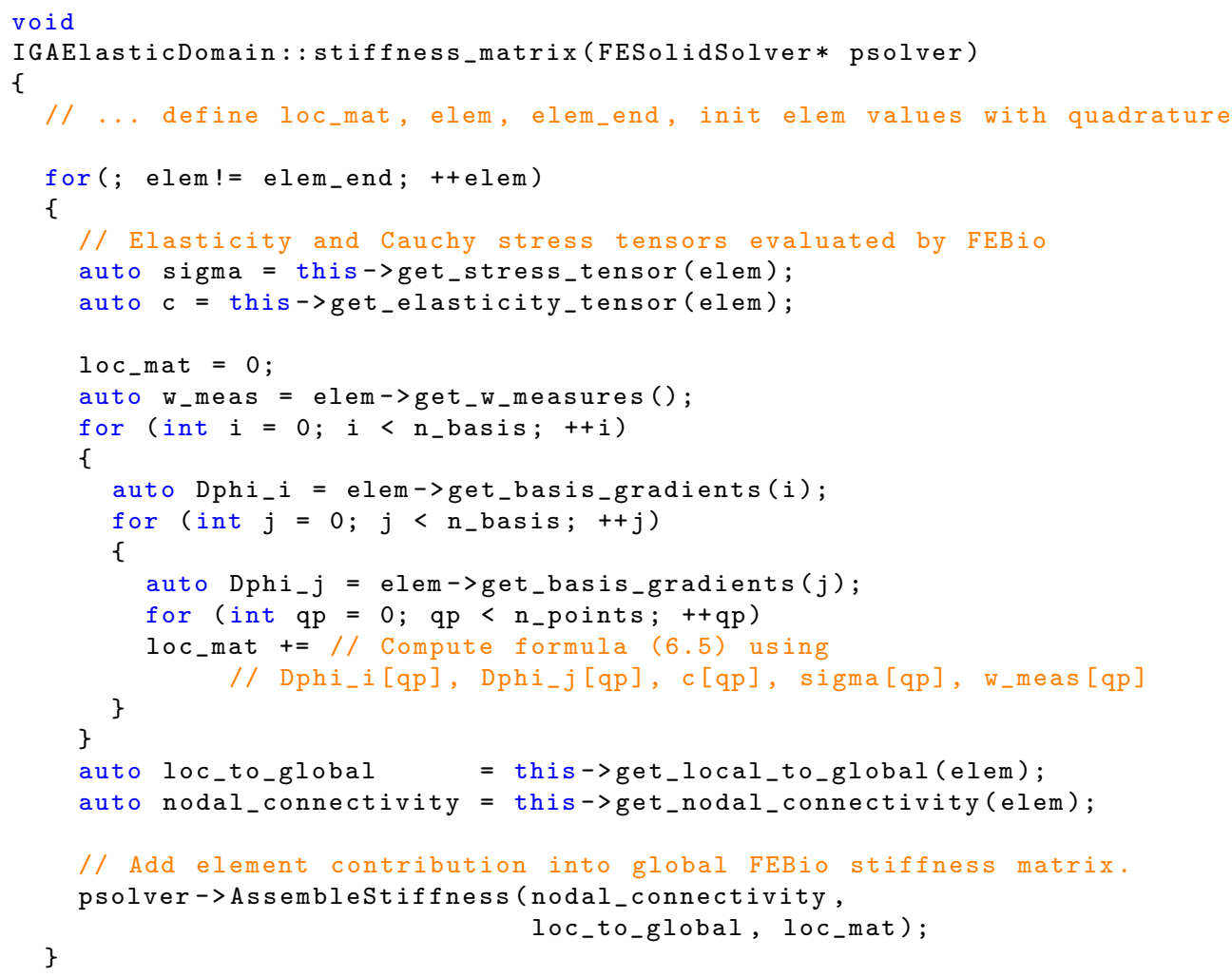

Implementation of the stiffness matrix for the nonlinear elasticity case. The local element matrices are assembled into the FEBio expected structure.

Finally, Figure 6.5 shows a simulation for the torsion of a curved tube with an open section. The geometry is given by a three dimensional single NURBS patch of degree two and its material modeled by a Neo-Hookean formulation.

Acknowledgements. We would like to thank A. Buffa and G. Sangalli for feedback and suggestions 


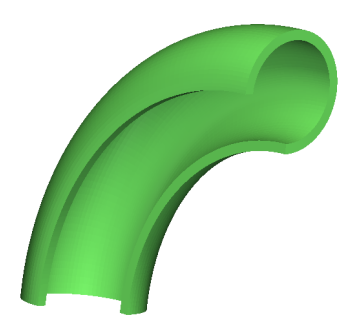

(a) The geometry map.

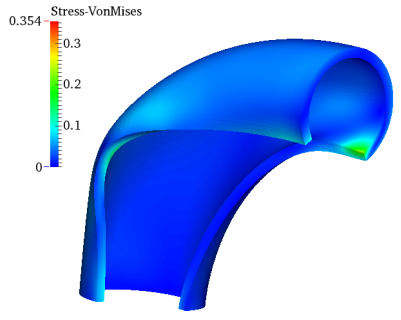

(b) $t=0.3$

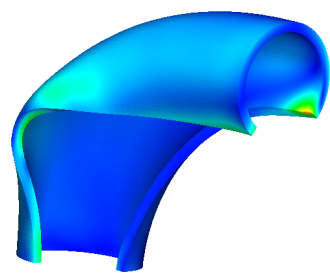

(c) $t=0.5$

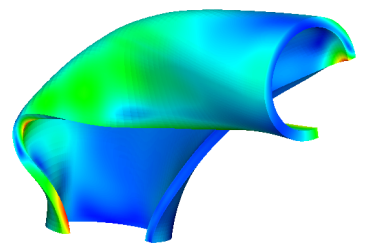

(d) $t=1.0$

FIG. 6.5. A non-linear elasticity simulation for an open tube is shown. The map is a second order deformation of a square 6.5(a), In this simulation a rotation around the axis of the tube is prescribed in one of the ends of tube whereas the other is fixed. Finite deformations are considered for an hyperelastic material formulation. Colormap represents von Mises stress.

and to the beta testers of igatools for their patience and feedback, in particular to A. Bressan, E. Brivadis, and R. Vázquez.

\section{REFERENCES}

[1] D. N. Arnold, R. S. Falk, and R. Winther, Finite element exterior calculus: from Hodge theory to numerical stability, Bull. Amer. Math. Soc. (N.S.), 47 (2010), pp. 281-354.

[2] W. Bangerth, R. Hartmann, and G. Kanschat, deal.II - a general purpose object oriented finite element library, ACM Trans. Math. Softw., 33 (2007), pp. 24/1-24/27.

[3] W. Bangerth, T. Heister, And G. Kanschat, deal. II Differential Equations Analysis Library, Technical Reference. http://www.dealii.org.

[4] Y. Bazilevs, L. Beirão da Veiga, J. A. Cottrell, T. J. R. Hughes, and G. Sangalli, Isogeometric analysis: Approximation, stability and error estimates for h-refined meshes, Math. Mod. Meth. Appl. S., 16 (2006), pp. 10311090.

[5] Y. Bazilevs, V. M. Calo, J. A. Cottrell, J. A. Evans, T. J. R. Hughes, S. Lipton, M. A. Scott, and T. W. Sederberg, Isogeometric analysis using T-splines, Comput. Methods Appl. Mech. Engrg., 199 (2010), pp. 229-263.

[6] Y. Bazilevs, C. Michler, V. M. Calo, and T. J. R. Hughes, Isogeometric variational multiscale modeling of wallbounded turbulent flows with weakly enforced boundary conditions on unstretched meshes, Comput. Methods Appl. Mech. Engrg., 199 (2010), pp. 780-790.

[7] Y. Bazilevs, C. Michler, V. M. Calo, and T. J. R. Hughes, Isogeometric variational multiscale modeling of wallbounded turbulent flows with weakly enforced boundary conditions on unstretched meshes, Comput. Methods Appl. Mech. Engrg., 199 (2010), pp. 780-790.

[8] K. Beck, Test Driven Development: By Example, Addison-Wesley Longman, 2002.

[9] P. BECKER, Working draft, standard for programming language C++, Tech. Report N3242=11-0012, ISO/IEC JTC 1, Information Technology, Subcommittee SC 22, Programming Language C++, Feb. 2011.

[10] D. J. Benson, Y. Bazilevs, E. De Luycker, M.-C. Hsu, M. Scott, T. J. R. Hughes, and T. Belytschko, A generalized finite element formulation for arbitrary basis functions: From isogeometric analysis to XFEM, Int. J. Numer. Meth. Eng., 83 (2010), pp. 765-785.

[11] D. Boffi, F. Brezzi, And M. Fortin, Mixed Finite Element Methods and Applications, Springer Series in Computational Mathematics, Springer London, Limited, 2013.

[12] J. Bonet And R. D. Wood, Nonlinear Continuum Mechanics for Finite Element Analysis, Cambridge University Press, New York, NY, USA, 2 ed., 2008.

[13] M. J. Borden, M. A. Scott, J. A. Evans, and T. J. R. Hughes, Isogeometric finite element data structures based on Bézier extraction of NURBS, Int. J. Numer. Meth. Eng., 87 (2011), pp. 15-47.

[14] A. Bressan And G. SAngaldi, Isogeometric discretizations of the Stokes problem: stability analysis by the macroelement technique, IMA J. Numer. Anal., (2012).

[15] A. Buffa, D. Cho, And G. Sangalli, Linear independence of the T-spline blending functions associated with some particular T-meshes, Comput. Methods Appl. Mech. Engrg., 199 (2010), pp. 1437-1445.

[16] A. Buffa, C. de Falco, and G. Sangalli, IsoGeometric Analysis: stable elements for the $2 D$ Stokes equation, Int. J. Numer. Meth. Fl., 65 (2011), pp. 1407-1422.

[17] A. Buffa, J. Rivas, G. Sangalli, And R. VÁzquez, Isogeometric discrete differential forms in three dimensions., SIAM J. Numer. Anal., 49 (2011), pp. 818-844.

[18] A. Buffa, G. Sangalli, And R. VÁzquez, Isogeometric analysis in electromagnetics: B-splines approximation, Com- 
put. Methods Appl. Mech. Engrg., 199 (2010), pp. 1143-1152.

[19] J. A. Cottrell, T. J. R. Hughes, And Y. Bazilevs, Isogeometric Analysis: Toward Integration of CAD and FEA, Wiley, 2009.

[20] J. A. Cottrell, A. Reali, Y. Bazilevs, and T. J. R. Hughes, Isogeometric analysis of structural vibrations, Comput. Methods Appl. Mech. Engrg., 195 (2006), pp. 5257-5296.

[21] C. de Falco, A. Reali, and R. Vázquez, GeoPDEs: A research tool for isogeometric analysis of PDEs, Adv. Eng. Softw., 42 (2011), pp. 1020 - 1034.

[22] T. Dokken, T. Lyche, and K. F. Pettersen, Locally refinable splines over box-partitions, tech. report, SINTEF, February 2012.

[23] M. Dörfel, B. Jüttler, And B. Simeon, Adaptive isogeometric analysis by local h-refinement with T-splines, Comput. Methods Appl. Mech. Engrg., 199 (2009), pp. 264-275.

[24] J. A. Evans And T. J. R. Hughes, Isogeometric divergence-conforming B-spline for the steady Navier-Stokes equations, Math. Mod. Meth. Appl. S., 23 (2013), pp. 1421-1478.

[25] _ Isogeometric divergence-conforming B-splines for the unsteady Navier-Stokes equations, J. Comput. Phys., 241 (2013), pp. 141-167.

[26] D. R. Forsey AND R. H. BARTELs, Hierarchical B-spline refinement, in SIGGRAPH '88 Proceedings of the 15th annual conference on Computer graphics and interactive techniques, 1988, pp. 205-212.

[27] C. Giannelli, B. Jütther, And H. Speleers, THB-splines: The truncated basis for hierarchical splines, Compute. Aided Geometric D., 29 (2012), pp. 485-498.

[28] R. Glowinski, P. G. Ciarlet, and J. L. Lions, Numerical Methods for Fluids, vol. 3 of Handbook of numerical analysis, Elsevier, 2002.

[29] M. Heroux, R. Bartlett, V. E. Howle, R. Hoekstra, J. Hu, T. Kolda, R. Lehoucq, K. Long, R. Pawlowski, E. Phipps, A. Salinger, H. Thornquist, R. Tuminaro, J. Willenbring, and A. Williams, An Overview of Trilinos, Tech. Report SAND2003-2927, Sandia National Laboratories, 2003.

[30] T. J. R. Hughes, J. A. Cottrell, And Y. Bazilevs, Isogeometric analysis: CAD, finite elements, NURBS, exact geometry and mesh refinement, Comput. Methods Appl. Mech. Engrg., 194 (2005), pp. 4135-4195.

[31] ISO/IEC 14882:2003 - Programming languages - C++, 2003.

[32] ISO/IEC 14882:2011 - Information technology - programming languages - $C++, 2011$.

[33] J. Kiendl, Y. Bazilevs, M.-C. Hsu, R. Wüchner, And K.-U. Bletzinger, Kirchhoff-Love shell structures comprised of multiple patches, Comput. Methods Appl. Mech. Engrg., 199 (2010), pp. 2403-2416.

[34] S. A. Maas, B. J. Ellis, G. A. Ateshian, and J. A. Weiss, FEBio: Finite elements for biomechanics, J. Biomed. Eng., 134 (2012).

[35] P. Monk, Finite element methods for Maxwell's equations, Numerical Mathematics and Scientific Computation, Oxford University Press, New York, 2003.

[36] L. A. Piegl And W. Tiller, The NURBs Book, Monographs in Visual Communication Series, Springer-Verlag GmbH, 1997.

[37] W. Schroeder, K. Martin, and B. Lorensen, The Visualization Toolkit: An Object-oriented Approach to 3D Graphics, Kitware, 2006.

[38] L. L. Schumaker, Spline functions: basic theory, Cambridge Mathematical Library, Cambridge University Press, Cambridge, third ed., 2007.

[39] M. A. Scott, X. Li, T. W. Sederberg, and T. J. R. Hughes, Local refinement of analysis-suitable T-splines, Comput. Methods Appl. Mech. Engrg., 213-216 (2012), pp. 206 - 222.

[40] A. H. Squillacote, The ParaView Guide, Kitware, 2007.

[41] A. Stepanov and M. Lee, The standard template library, tech. report, WG21/N0482, ISO Programming Language $\mathrm{C}++$ Project, 1994

[42] B. Stroustrup, The C++ Programming Language, Addison-Wesley, 2000.

[43] A.-V. Vuong, C. Giannelli, B. Jüttler, And B. Simeon, A hierarchical approach to adaptive local refinement in isogeometric analysis, Comput. Methods Appl. Mech. Engrg., 200 (2011 Dec), pp. 3554-3567.

[44] O. WeEger, U. Wever, AND B. Simeon, Isogeometric analysis of nonlinear Euler Bernoulli beam vibrations, Nonlinear Dynam., 72 (2013), pp. 813-835. 